\title{
Error reduction for time-resolved PIV data based on Navier-Stokes equations
}

\author{
Hong-Ping Wang ${ }^{1} \cdot \mathrm{Qi} \mathrm{Gao}^{2}{ }^{-1} \cdot$ Shi-Zhao Wang ${ }^{1} \cdot$ Yu-Hang $\mathrm{Li}^{1} \cdot$ Zhong-Yi Wang $^{3} \cdot$ Jin-Jun Wang ${ }^{3}$
}

Received: 7 January 2018 / Revised: 7 July 2018 / Accepted: 31 August 2018 / Published online: 12 September 2018

(c) Springer-Verlag GmbH Germany, part of Springer Nature 2018

\begin{abstract}
The post-processing of the measured velocity in particle image velocimetry (PIV) is a critical step in reducing error and predicting missing information of the flow field. In this work, time-resolved PIV data are incorporated with the incompressible Navier-Stokes (N-S) equations to reduce the measurement error and improve the accuracy. A pressure correction scheme (PCS) based on the projection method is adopted to solve the $\mathrm{N}-\mathrm{S}$ equations, and an optimization algorithm is introduced to balance the fidelity between the PIV data and the numerical solutions. The PCS for PIV data, called PIV-PCS, cannot only reduce the errors in the velocity divergence and the curl of the pressure gradient but also ensure that the flow field satisfies the dynamic constraints imposed by the momentum equation. An important weight coefficient $s$ that balances the level of the velocity modification with the residual of the governing equation is defined and numerically assessed. A method for optimizing the value of $s$ is provided. The new approach is evaluated by two time-resolved PIV experiments: one on the 2D wake flow of a circular cylinder at low Reynolds number and one on tomographic PIV for the 3D wake flow of a hemisphere at high Reynolds number. All the numerical assessments and experimental applications are compared with the divergencefree smoothing (DFS) method. The results indicate that the presented PIV-PCS method is superior to the DFS method in terms of reducing the measurement error and recovering the real physical flow structures.
\end{abstract}

\section{Introduction}

Particle image velocimetry (PIV) is regarded as the most reliable non-invasive technique to quantitatively measure the motion of flows. With the development of tomographic PIV (Elsinga et al. 2006), three-dimensional (3D) flow fields can be obtained with high accuracy. The PIV technique has been widely used in the field of experimental fluid mechanics. However, people want to access more information about flows from PIV, such as pressure, density and temperature, and not simply velocity. Meanwhile, since the velocity is deduced from the particle intensity distribution in 2D images or 3D volumes by cross-correlation or particle tracking algorithms, PIV measurements often suffer from various sources

Qi Gao

qigao@zju.edu.cn

1 State Key Laboratory of Nonlinear Mechanics, Institute of Mechanics, Chinese Academy of Sciences, Beijing, China

2 School of Aeronautics and Astronautics, Zhejiang University, Hangzhou, China

3 Key Laboratory of Fluid Mechanics (Beihang University), Ministry of Education, Beijing, China of experimental error such as insufficient tracer particles, out-of-plane issues of particle motion and ghost particles in tomographic PIV. The errors in the velocity field not only affect the identification of flow structures but also can propagate into the calculated pressure, acceleration and other flow quantities (Andrea and Bernhard 2016). Therefore, error reduction and missing information prediction (such as pressure, density and temperature) are of important significance for PIV community.

This paper only focuses on the error reduction in PIV. Therefore, available error reduction methods are summarized in Table 1, and they are classified into three categories: mathematical models, kinematic models and physical models. This classification strategy is based on the implementation of error reduction, not limited to post-processing. Moreover, methods listed in Table 1 are not only for reducing measurement error, they can also serve for achieving other purposes. Error reduction by post-processing methods usually consist of data validation, interpolation and smoothing (Garcia 2011). Data validation is applied to identify the outliers, where one of the most popular methods for data validation in PIV software is the normalized median test proposed by Westerweel and Scarano (2005). After the 


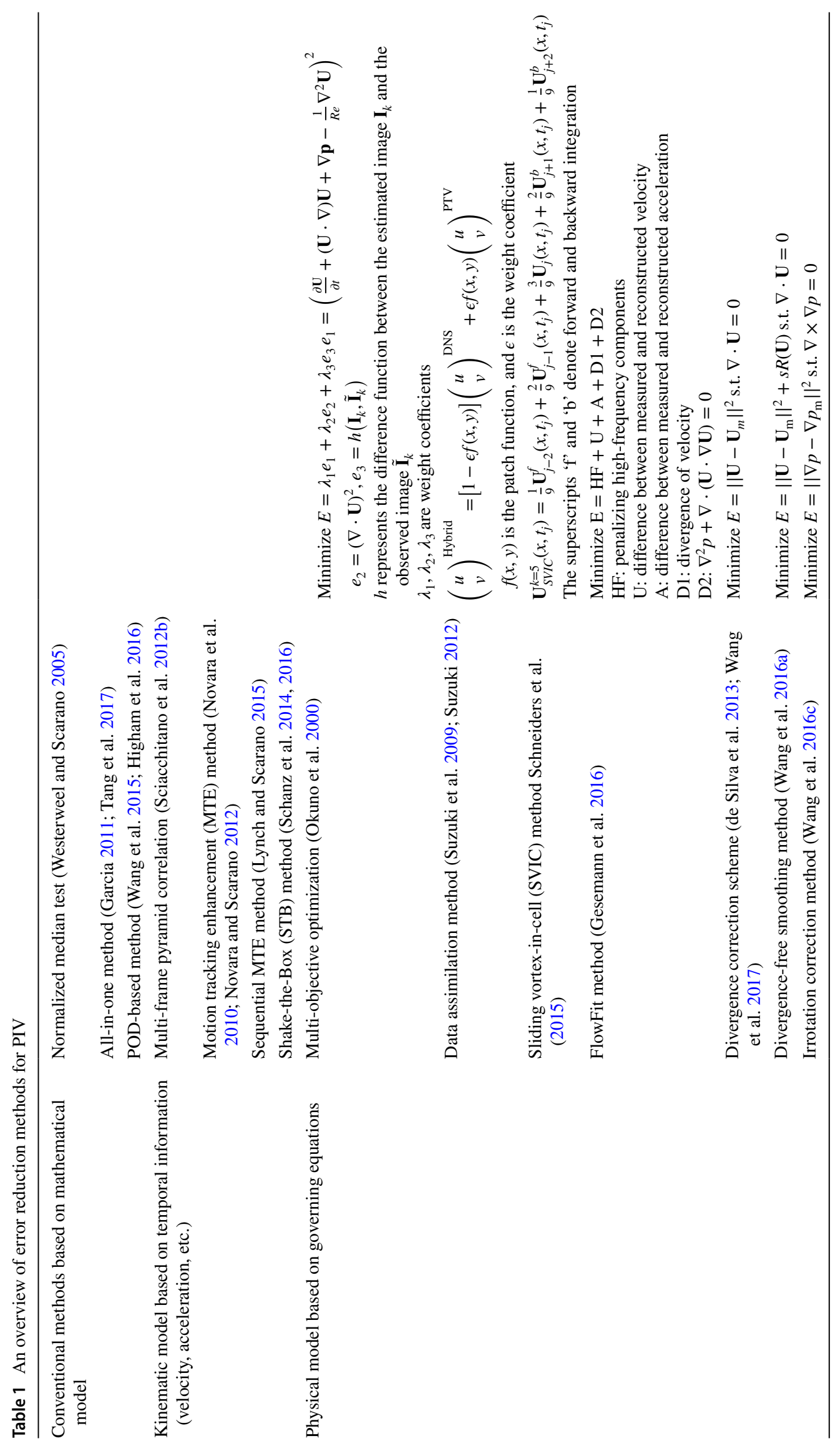


outliers are detected, the incorrect and missing velocities need to be replaced by a local mean or linear, spline or Kriging interpolations (Gunes and Rist 2007). Data smoothing is usually adopted in the final step to reduce the random noise in PIV measurements. This is achieved by convolving the velocity field with a proper filter kernel (Raffel 2007). To improve the efficiency of post-processing, Garcia (2011) proposed a robust post-processing technique that performs all the steps simultaneously. This method combines a penalized least squares approach (PLS) and the discrete cosine transform (DCT) to validate and smooth the PIV data, called DCT-PLS or the all-in-one method. Based on DCT-PLS, a vector field correction method was proposed to automatically classify the flows with scattered and clustered outliers and to treat them in a discriminatory manner (Tang et al. 2017). Proper orthogonal decomposition (POD) is another effective approach to restoring gaps in data (Venturi and Karniadakis 2004), and detecting and replacing spurious vectors (Wang et al. 2015; Higham et al. 2016). Although conventional methods normally achieve good performances in terms of handling errors in PIV data in a mathematical manner, they unfortunately do not satisfy the physical laws of flow motions, which could be either the kinematics or dynamics of fluids.

For time-resolved PIV (TR-PIV) data, the temporal information of the image sequences regarding particle kinematics can be used to reduce both the random and bias errors. These methods are normally embedded in the stage of raw velocity estimation. Sciacchitano et al. (2012b) proposed a multi-frame pyramid correlation that combines the correlation maps of different temporal separations to enhance the precision of cross-correlations. For the multiplicative algebraic reconstruction technique (MART) of tomographic PIV, a motion tracking enhancement MART (MTE-MART) method was proposed to improve the reconstructed particle intensity fields by eliminating suspicious particles who could not be tracked among multiple exposures (Novara et al. 2010; Lynch and Scarano 2015); such particles are normally considered as ghost particles. It was found that this method is more effective than MART in terms of computational cost reduction and ghost particle suppression. Recently, an advanced particle tracking velocimetry (PTV), called the shake-the-Box (STB) scheme (Schanz et al. 2014, 2016), was used to obtain high-resolution velocity fields for volumetric flow measurements. The STB method uses the temporal information to improve the precision of locating particles. Therefore, this method enables the processing of densely seeded flows on a similar order of seeding density within the tomographic PIV measurements (Schneiders and Scarano 2016).

Regarding fluid dynamics, the governing equations, especially the $\mathrm{N}-\mathrm{S}$ equations, can be considered as potential physical constraints for displacement estimation or the post-processing of PIV/PTV data. Given the constraint from the governing equations, certain unmeasured quantities can be deduced from velocity fields. For instance, the unmeasured component of the velocity gradient tensor $\partial w / \partial z$ can be directly calculated from the incompressible continuity equation in a dual-plane PIV measurement (Ganapathisubramani et al. 2005, 2006). Another successful application is to access the unmeasured pressure field by solving the incompressible momentum equation (Liu and Katz 2006; John et al. 2010; Oudheusden 2013). Along these lines, Sciacchitano et al. (2012a) recovered the missing velocity field of PIV measurements directly by solving the $\mathrm{N}-\mathrm{S}$ equations. The authors used an incompressible finite volume $\mathrm{N}-\mathrm{S}$ solver to fill the gappy data by imposing boundary conditions (BCs) from PIV data. The results indicated that the accuracy of this method is higher than that of mathematical interpolations.

In addition to the prediction of flow information, the other general applications of the governing equations are in reducing measurement error or recovering spatial/temporal details by forcing the governing laws to constrain the experimental data. Okuno et al. (2000) constructed a multi-objective optimization problem that consists of the residues of the momentum equation, the continuity equation and the difference between the estimated and observed image data to solve the velocity. They used an evolutionary programming (EP) to obtain the solutions. Suzuki et al. (2009) developed a hybrid unsteady-flow simulation technique by combining PTV and direct numerical simulation (DNS). As a result, the reconstructed velocity field can satisfy the governing equations with a fine-resolution compared to numerical simulation (Suzuki et al. 2009; Suzuki 2012). However, this hybrid simulation needs to be performed on the full computational mesh, which is the same as the mesh used in DNS. The vortex-in-cell (VIC) method proposed by Schneiders et al. (2014) uses the vorticity transport equation to increase the temporal resolution of a TR-PIV measurement. Its advanced version, namely the VIC+ method, optimizes for $\mathrm{BCs}$ and vorticity field to fit the vorticity transport equation and the measurement data, which are adopted to reconstruct dense velocity of volumetric measurements (Schneiders and Scarano 2016). With the sliding VIC method, multiple instantaneous measurements at a single time instant are achieved by both backward and forward time integrations with the VIC simulation. Averaging the multiple repeatedly simulated measurements can reduce the random noise without the effect produced by spatial and temporal smoothing (Schneiders et al. 2015). A B-spline-based method called 'FlowFit' and proposed by Gesemann et al. (2016) uses a joint optimization of divergence and acceleration as physical constraints to recover spatial details and pressure as well as to reduce noise. The weighting factors in the FlowFit method need to be determined based on the flow properties and measurement qualities (Schneiders and Scarano 2016). Moreover, part of 
the N-S equations can also be used to optimize the PIV or PTV data. The divergence-free condition is used to reduce the noise in PIV measurements (de Silva et al. 2013; Wang et al. 2016a, 2017), and an irrotation correction (Wang et al. 2016c) is applied to the pressure gradient to improve the accuracy of the pressure estimation.

As listed in Table 1 , the $\mathrm{N}-\mathrm{S}$ equation has already been adopted to determine the velocity from noisy measurement data by Okuno et al. (2000) and Schneiders et al. (2015). In this work, we will explore the suitability of solving the N-S equations in the measurement domain using the measured BCs. For combining the numerical solver and noisy PIV data, a new optimization function is deduced from the projection method of computational fluid dynamics (CFD). The robustness of this method to noise is discussed in detail. Two experimental applications are considered: a 2D flow around a circular cylinder at low Reynolds number and a 3D wake flow of a hemisphere with a laminar boundary layer.

\section{Working principle}

The working principle of error reduction for TR-PIV data based on $\mathrm{N}-\mathrm{S}$ equations will be introduced for the case of a two-dimensional incompressible flow for simplicity. The 3D case will be analyzed in the experimental applications. Under the two-dimensional hypothesis, this method is appropriate only if the out-of-plane component is negligible with respect to the other two components in the measured plane. A detailed discussion about the two-dimensional hypothesis can be found in the paper by Sciacchitano et al. (2012a). The two components lying within the measured plane are denoted by $u$ and $v$, corresponding to the horizontal $x$ direction and the vertical $y$ direction.

\subsection{The pressure correction scheme for projection method}

The N-S equations in non-dimensional conservation form for two dimensions (2D) read

$$
\begin{aligned}
u_{t}+\left(u^{2}\right)_{x}+(u v)_{y} & =-p_{x}+\frac{1}{R e}\left(u_{x x}+u_{y y}\right) \\
v_{t}+(u v)_{x}+\left(v^{2}\right)_{y} & =-p_{y}+\frac{1}{R e}\left(v_{x x}+v_{y y}\right) \\
u_{x}+v_{y} & =0 .
\end{aligned}
$$

In this equation, the velocity $(\mathbf{U}=[u, v])$ and coordinate $(\mathbf{X}=[x, y])$ are scaled by a reference velocity $U_{r e f}$ and length $L_{\text {ref }}$. Thus, the time is normalized by $L_{\text {ref }} / U_{\text {ref }}$, the pressure $p$ is scaled by $\rho U_{\text {ref }}^{2}$, and the Reynolds number $R e$ is equal to $\rho U_{\text {ref }} L_{\text {ref }} / \mu$. The parameters $\rho$ and $\mu$ are the density and dynamic viscosity coefficient, respectively. The subscript represents the partial derivative with respect to time $t$ or the $x$ and $y$ directions.

Projection methods are widely adopted to solve the N-S equations. These methods were proposed in the late 1960s in the ground-breaking work of Chorin (1968) and Témam (1969). The most attractive feature of projection methods is that, at each time step, one only needs to solve a sequence of decoupled elliptic equations for the velocity and pressure (Guermond et al. 2006). Moreover, an optimization algorithm can easily be introduced into the linearized equation of the velocity. The pressure correction scheme, hereafter abbreviated PCS, is a time-marching technique adopted in projection methods (Guermond et al. 2006). Comprehensive descriptions of the numerical method are given in the paper by Guermond et al. (2006) and the book by Strang (2007). In this work, we will make a brief introduction to the original PCS. In PCS, the $\mathrm{N}-\mathrm{S}$ equation is decomposed into three terms: the nonlinear term (Eq. 2), the viscosity term (Eq. 3) and the pressure correction term (Eq. 4):

$$
\begin{aligned}
& \frac{u^{*}-u^{k}}{\mathrm{~d} t}=-\left(u^{k} \cdot u^{k}\right)_{x}-\left(u^{k} \cdot v^{k}\right)_{y}-p_{x}^{k} \\
& \frac{v^{*}-v^{k}}{\mathrm{~d} t}=-\left(u^{k} \cdot v^{k}\right)_{x}-\left(v^{k} \cdot v^{k}\right)_{y}-p_{y}^{k}, \\
& \frac{u^{* *}-u^{*}}{\mathrm{~d} t}=\frac{1}{\operatorname{Re}}\left(u_{x x}^{* *}+u_{y y}^{* *}\right) \\
& \frac{v^{* *}-v^{*}}{\mathrm{~d} t}=\frac{1}{\operatorname{Re}}\left(v_{x x}^{* *}+v_{y y}^{* *}\right),
\end{aligned}
$$

$\frac{u^{k+1}-u^{* *}}{\mathrm{~d} t}=-p_{x}^{k+1}+p_{x}^{k}$
$\frac{v^{k+1}-v^{* *}}{\mathrm{~d} t}=-p_{y}^{k+1}+p_{y}^{k}$.

The solution at the $k+1$ time step is estimated in the following three steps:

Step 1 Calculate the intermediate variable $\mathbf{U}^{*}$ in the explicit nonlinear term as Eq. 2. The velocity field $\mathbf{U}^{\mathbf{k}}$ and pressure field $p^{k}$ have been solved from the previous step.

Step 2 Solve the intermediate variable $\mathbf{U}^{* *}$ in the implicit viscous term as Eq. 3 by constructing a linear equation set. If we use a square matrix $\mathbf{L}_{u}$ and $\mathbf{L}_{v}$ to replace the Laplace operator of $u$ and $v$, this equation can be rewritten in matrix form:

$$
\begin{aligned}
& \left(\mathbf{I}-\frac{\mathrm{d} t}{R e} \mathbf{L}_{u}\right) \mathbf{u}^{* *}=\mathbf{u}^{*} \\
& \left(\mathbf{I}-\frac{\mathrm{d} t}{\operatorname{Re}} \mathbf{L}_{v}\right) \mathbf{v}^{* *}=\mathbf{v}^{*}
\end{aligned}
$$

where I is the identity matrix. An implicit treatment of the viscous term can relax the restriction on the time separation $\mathrm{d} t$. 
Step 3 Solve the Poisson Eq. 6. This equation is obtained by applying the divergence-free condition to the velocity field $\mathbf{U}^{\mathbf{k}+\mathbf{1}}$. Thus, the pressure correction Eq. 4 can be rewritten as

$$
\Delta p^{k+1}=\Delta p^{k}+\frac{1}{\mathrm{~d} t} \nabla \cdot \mathbf{U}^{* *} .
$$

Here, $\nabla \cdot \mathbf{U}^{* *}$ is the divergence of the intermediate variable calculated from Eq. 3. Both of the terms on the right-hand side of Eq. 6 are known, and this equation can also be written as $\mathbf{L}_{p} p^{k+1}=b^{* *}$ if the Laplace operator is replaced by a square matrix $\mathbf{L}_{p}$ and the terms on the right-hand side are replaced by $b^{* *}$. After the pressure $p^{k+1}$ is calculated by solving the Poisson equation, the velocity field is updated:

$u^{k+1}=u^{* *}-\mathrm{d} t \cdot\left(p_{x}^{k+1}-p_{x}^{k}\right)$

$v^{k+1}=v^{* *}-\mathrm{d} t \cdot\left(p_{y}^{k+1}-p_{y}^{k}\right)$.

The velocity field $\mathbf{U}^{\mathbf{k}+\mathbf{1}}$ can gradually satisfy the divergencefree condition after this correction.

The $\mathbf{U}^{*}$ and $\mathbf{U}^{* *}$ are the intermediate variables of the velocity field, and the parameter $d t$ is the time step. In this context, the time derivative is approximated by a backward difference formula of first order (BDF1), and the terms on the left-hand side of Eqs. 2-4 can also be replaced by a highorder BDF or the more popular Adams schemes (Guermond et al. 2006). These Poisson equations (Eqs. 5 and 6) can be easily and efficiently solved by applying a sparse Cholesky decomposition.

\subsection{Optimization with the PIV field}

PIV data are always contaminated by varying degrees of noise. It is intuitive and logical to optimize the PIV velocity field by introducing physical constraints imposed by the N-S equations. We propose a modified PCS combined with PIV data (PIV-PCS) to optimize the PIV field. Figure 1 shows the flowchart of PIV-PCS optimization algorithm. From the equations listed in Fig. 1, we can clearly see the differences between PIV-PCS and original PCS, and these differences will be introduced in this section.

For TR-PIV, the PIV-based pressure field $p_{m}$ can be calculated by the Poisson equation or linear integration approaches (Liu and Katz 2006) from the measured velocity field $\mathbf{U}_{m}$, the subscript ' $m$ ' denotes measurement. Instead of the numerical solution $p^{k}$ from the previous step for the original PCS, in PIV-PCS, we use the estimated pressure $p_{m}^{k+1}$ at the $k+1$ time step as the initial pressure for Eqs. 2 and 4 . The modified equations can be found in Fig. 1 of flowchart of PIV-PCS. We do not use the pressure gradient deduced from the PIV data because the error in the pressure gradient can be amplified by the time derivative. If we accept

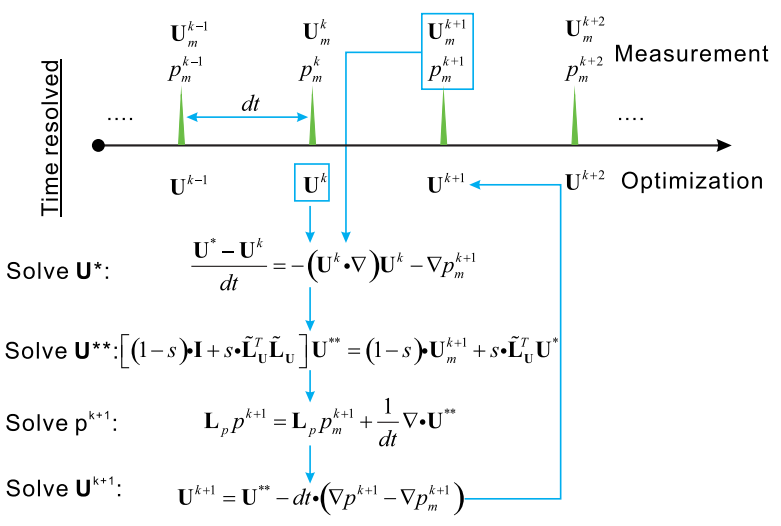

Fig. 1 The diagram for PIV-PCS method. The equations are given in matrix form for brevity

the assumption that the PIV data are sufficiently accurate to provide the $\mathrm{BCs}$, it is reasonable to conclude that the numerical solution of the pressure $p^{k+1}$ in Eq. 4 is equal to $p_{m}^{k+1}$. Substitute this conclusion into Eq. 7 ( $p^{k}$ is already replaced by $p_{m}^{k+1}$ ), it will be proved that the numerical solution $\mathbf{U}^{* *}=\mathbf{U}_{m}^{k+1}$ when the PIV data are free of noise.

Considering the measurement error, there are two significant problems when numerically solving the velocity and pressure in the measurement domain by imposing $\mathrm{BCs}$ and initial conditions from the PIV data. First, the error in the measured velocity fields will propagate from the PIV data to the numerical solutions. Second, the time separation is always larger than the requirement of numerical simulation, this will result in a temporal truncation error for time derivative. The influence of the temporal truncation, however, is much smaller than the measurement error propagation. Moreover, PIV-based pressure measurement errors are dominated by spatial modulation and not by temporal truncation errors (Lynch et al. 2014; van Gent et al. 2017). Thus, the optimization in PIV-PCS is mainly achieved by reducing the measurement error propagation. This technique consists of minimizing a criterion that balances the fidelity of the PIV data with a penalty term that corresponds to the numerical solution. According to the method proposed by Garcia (2010), one thus seeks to minimize the cost function of $u$ :

$J_{u}=\frac{1-s}{N} \sum_{\mathbf{X}}\left\|\mathbf{u}^{* *}-\mathbf{u}_{m}^{k+1}(\mathbf{X})\right\|^{2}+\frac{s}{N} \sum_{\mathbf{X}}\left\|\left(\mathbf{I}-\frac{\mathrm{d} t}{R e} \mathbf{L}_{u}\right) \mathbf{u}^{* *}-\mathbf{u}^{*}\right\|^{2}$,

where $\|\cdot\|$ denotes the $L_{2}$ norm. $\mathbf{u}^{* *}$ is the unknown velocity field, which will be solved by minimizing $J_{u}$. The cost function of $v$ has the same form as $J_{u}$. $\mathbf{u}_{m}^{k+1}$ is the velocity at $k+1$ time step obtained by the PIV measurement, and the parameter $N$ is the grid number of the computational mesh. $\mathbf{X}$ is the location of the simulation grid, and $\mathbf{u}^{*}$ is the temporary 
result obtained from Eq. 2. The parameter $s$ is a real positive scalar that controls the weight of the numerical solution: as $s$ increases from 0 to 1 , the numerical component in $\mathbf{u}^{* *}$ also increases. The first term on the right-hand side represents the residual sum of squares between the solution and the PIV data, in which $\mathbf{u}_{m}^{k+1}(\mathbf{X})$ denotes the PIV field interpolated on the computational grid. In this work, a highly accurate spline interpolation is adopted. The second term on the right-hand side is the penalty term of Eq. 5 .

The minimization of $J_{u}$ and $J_{v}$ yields the following linear system that can be used to update the $\mathbf{u}^{* *}$ and $\mathbf{v}^{* * *}$ :

$$
\begin{aligned}
& {\left[(1-s) \cdot \mathbf{I}+s \cdot \widetilde{\mathbf{L}}_{u}^{\mathrm{T}} \widetilde{\mathbf{L}}_{u}\right] \mathbf{u}^{* *}=(1-s) \cdot \mathbf{u}_{m}^{k+1}+s \cdot \widetilde{\mathbf{L}}_{u}^{\mathrm{T}} \mathbf{u}^{*}} \\
& {\left[(1-s) \cdot \mathbf{I}+s \cdot \widetilde{\mathbf{L}}_{v}^{\mathrm{T}} \widetilde{\mathbf{L}}_{v}\right] \mathbf{v}^{* *}=(1-s) \cdot \mathbf{v}_{m}^{k+1}+s \cdot \widetilde{\mathbf{L}}_{v}^{\mathrm{T}} \mathbf{v}^{*}}
\end{aligned}
$$

Here, $\widetilde{\mathbf{L}}_{u}$ and $\widetilde{\mathbf{L}}_{v}$ are equal to $\mathbf{I}-\frac{\mathrm{d} t}{R e} \mathbf{L}_{u}$ and $\mathbf{I}-\frac{\mathrm{d} t}{R e} \mathbf{L}_{v}$, respectively. The superscript ' $\mathrm{T}$ ' stands for the transpose. The difference between $\widetilde{\mathbf{L}}_{u}$ and $\widetilde{\mathbf{L}}_{v}$ is caused by the application of a staggered mesh, which will be introduced in Sect. 2.3. Equation 9 is used to replace Eq. 5. In these linear systems, $\mathbf{u}^{* *}$ and $\mathbf{v}^{* *}$ are the only unknown variables if the parameter $s$ is given.

The weight coefficient $s$ is in the range of $0 \leq s \leq 1$. When $s$ increases from 0 to 1, the result of Eq. 9 shifts from measurement to numerical simulation. Even if the parameter $s$ is forced to be 1 , the uncorrected BCs may contaminate the numerical solutions. Moreover, the high wave-number measurement error may be amplified by the nonlinear operator (Suzuki et al. 2006, 2009). In the paper by Suzuki et al. (2009), a linear combination between DNS and PTV data was proposed to achieve a high-fidelity simulation. They used the linearized $\mathrm{N}-\mathrm{S}$ equation to analyze the stability of the hybrid algorithm when the time separation $d t$ is small. In this context, we adopt the suggestion that the $s$ should satisfy

$s \leq 1-\alpha \mathrm{d} t\left|\frac{\partial v}{\partial x}-\frac{\partial u}{\partial y}\right|_{\max }$,

so that the error will not be amplified under small $\mathrm{d} t$. This equation is deduced from the Kelvin-Helmholtz instability of a shear layer, and the coefficient $\alpha$ is approximately 0.2 for a planar shear layer in the paper by Suzuki et al. (2009). The maximum vorticity in Eq. 10 is calculated from the mean velocity field excluding the boundaries. In this context, we propose that $\alpha$ should be approximately ten for the PIV-PCS method from the numerical assessments, which will be introduced in Sect. 3.1.

The optimized velocity field can be obtained by substituting $\mathbf{U}^{* *}$ from Eq. 9 into Eqs. 6 and 7. We must focus on the pressure solved from Eq. 6 whose source terms contain the pressure and divergence of the measured velocity (Suzuki et al. 2009). The solutions obtained for pressure is used to satisfy the divergence-free condition, which implies that the pressure solved from Eq. 6 is not the real physical pressure when the velocity field is contaminated by errors.

\subsection{The staggered mesh and discretization method}

In the field of CFD, a staggered mesh, as shown in Fig. 2, is a classical scheme to overcome numerical instabilities. Normally, $u, v$ and $p$ are defined at different points, the pressure $p_{i, j}$ is located at the center of the $(i, j)$ cell, the horizontal velocity $u_{i, j}$ is placed on the right vertical cell interfaces, and the vertical velocity $v_{i, j}$ is placed on the above horizontal cell interfaces. The original points of $u, v$ and $p$ are located at the bottom-left corner of the computational domain, and their positions are different, as shown in Fig. 2b. The variables at the boundary points are unknown, and the method of applying the boundary conditions will be introduced in Sect. 2.4.

The spatial discretization can be conveniently performed on the staggered grid. According to Sect. 2.1, the N-S equation is decomposed into three types of terms: nonlinear terms, viscosity terms and pressure correction terms, and solved step by step. From Eq. 2 , it is obvious that $\left(u^{2}\right)_{x}$ and $(u v)_{y}$ should be defined at the same points as $u$, and $(u v)_{x}$ and $\left(v^{2}\right)_{y}$ should be defined at the same points as $v$. Moreover, the product $u v$ cannot be directly calculated since $u$ and $v$ are defined at different points in the staggered grid. The method for addressing the spatial discretization on the staggered grid can be found in the book by Strang (2007).

The classical CFL-type stability conditions must be satisfied to guarantee the numerical stability. The classical CFL number, which is given in Eq. 11, represents the ratio between the maximum displacement and the grid node

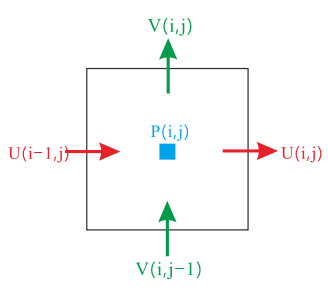

(a)

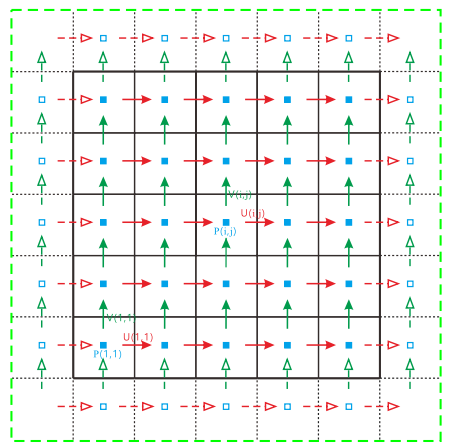

(b)
Fig. 2 a The $u, v$ and $p$ grid in a cell; $\mathbf{b}$ the diagram of the staggered mesh. The thick black line represents the edge of the computational domain, and the red and blue vectors represent the horizontal velocity $u$ and vertical velocity $v$, respectively. Any point truly inside the domain is an interior point, whereas points on or outside the boundaries are boundary points. For the velocity, the solid arrows represent the velocity components at the interior points, and the dashed arrows represent the velocity components at the boundary points. For the pressure, the solid squares indicate interior points, and the hollow squares indicate boundary points 
spacing ( $\mathrm{d} x$ and $\mathrm{d} y$ ). Generally, the CFL number should satisfy the stability condition and must be at least less than one. If we apply the PIV-PCS method to optimize the PIV data, the CFL condition must be considered before performing the experiment because the time separation between adjacent velocity snapshots and grid node spacing is determined by the configuration of the PIV system.

$\mathrm{CFL}=\max \left(\frac{\max (\mathbf{u}) \cdot \mathrm{d} t}{\mathrm{~d} x}, \frac{\max (\mathbf{v}) \cdot \mathrm{d} t}{\mathrm{~d} y}\right)<1$.

\subsection{The boundary conditions}

The above spatial discrete schemes only work for the interior points of the staggered mesh, and the finite-difference equations need to be addressed in a special manner to satisfy the boundary conditions. From the discussion in Sect. 2.1, we know that the pressure calculated from Eq. 6 is identical to the measured pressure if the PIV data are accurate. This implies that the intermediate variables $\mathbf{U}^{* *}$ are equal to $\mathbf{U}^{\mathbf{k}+\mathbf{1}}$ from Eq. 7. Therefore, it is natural to impose Dirichlet conditions on Eq. 9 by interpolating the PIV velocity field to the boundary points.

The Dirichlet condition of the pressure at a corner of the domain and the Neumann condition (pressure gradient) on the boundary nodes are enforced to the Poisson equation for pressure. The pressure gradient is estimated from the TRPIV measurements by $\mathrm{N}-\mathrm{S}$ equations. The boundary points (the thick black lines shown in Fig. 2) are not located at the center of the cells. Thus, the ghost cells (the cells outside the boundary) are used to discrete the difference equation on the boundary nodes.

Note that the influence of the PIV error on the BCs must be emphasized. Especially for the pressure estimation, a small velocity error can be magnified by an order of magnitude with a small time separation when deducing the pressure gradient from the $\mathrm{N}-\mathrm{S}$ equations at the boundary. Thus, the velocity on the boundary needs to be filtered beforehand. In the paper by John et al. (2010), three methods are proposed to smooth the velocity field. We adopt a PODbased smoothing method to filter the boundary velocity in this paper. The most energetically significant modes are used as the physical-based filter, and the last few modes, contributing less than $1 \%$ of the total energy, are removed as noise modes. To make a comparison, BCs with raw PIV data are also adopted. These two types of BCs are referred to as BCs-POD and BCs-Raw. Note that we only use the POD method to obtain the filtered boundary conditions; the interior velocity remains as raw PIV data. An effective way to address the BCs proposed by Schneiders and Scarano (2016) is to find the optimal BCs using an optimization procedure such as the VIC+ method.

\subsection{Working procedure}

The proposed method can be applied to both the 2D and 3D data of TR-PIV measurements. Based on numerical assessments, which will be shown in the next section, the PIV-PCS cannot reduce the modulation error caused by the interrogation window; therefore, the present work primarily concentrates on reducing the measurement noise.

Before substituting the PIV data into the numerical equations, the PIV data need to be normalized by reference velocity and length scales. It is noteworthy that the time in the numerical equations is a non-dimensional variable, not a real time. The proposed method has no other restriction on the spatial resolution except for the limitation of the CFL number.

Figure 1 shows the flowchart of PIV-PCS optimization algorithm, which consists of the following steps:

Step 1 The initial TR measured velocity field $\mathbf{U}_{m}$, which is free from large amplitude errors, such as outliers, is filtered by a POD-based smoothing method, as introduced in Sect. 2.4. The filtered velocity field is prepared to calculate the BCs.

Step 2 The initial pressure field $p_{m}$ is estimated from the raw PIV data and BCs. The pressure field is calculated on the PIV grid, and a second-order central-difference scheme is adopted for both the time derivative and the spatial gradient.

Step 3 The staggered mesh is generated according to the measurement domain. The computational domain is made smaller than the measurement domain to avoid boundary errors in the measurement. The numerical operators $\widetilde{\mathbf{L}}_{u}$, $\widetilde{\mathbf{L}}_{v}$ and $\mathbf{L}_{p}$ are generated beforehand into sparse matrices because these operators are invariant for each discrete time instant.

Step 4 The intermediate velocity $\mathbf{U}^{*}$ is solved on the staggered mesh from the velocity field $\mathbf{U}^{k}$ using Eq. 2 . Note that the pressure $p^{k}$ in Eq. 2 has been replaced by $p_{m}^{k+1}$. At the first time instant, the $\mathbf{U}^{1}$ is initialized by the measurement velocity.

Step 5 The optimized intermediate velocity $\mathbf{U}^{* *}$ is solved using Eq. 9. The parameter $s$ is determined using the condition of Eq. 10.

Step 6 The optimized velocity $\mathbf{U}^{k+1}$ is calculated from Eq. 7 after solving the pressure using Poisson's Eq. 6. Note that the pressure $p^{k}$ in Eqs. 6 and 7 has been replaced by $p_{m}^{k+1}$.

Step 7 Repeat steps 4-6 until all the PIV data are post-processed.

\section{Numerical assessments}

To assess the PIV-PCS algorithm, a 2D flow around a circular cylinder is simulated using an immersed boundary method based on the discrete stream function formulation developed 
by Wang and Zhang (2011). The numerical domain spans $-4 D \leq x \leq 20 D$ and $-10 D \leq y \leq 10 D$, with the cylinder being centered at the origin $(0,0)$, as shown in Fig. 3. The diameter of the circular cylinder $D$ is equal to $10 \mathrm{~mm}$. The normalized $\mathrm{N}-\mathrm{S}$ equation is solved on a $2 \mathrm{D}$ uniform mesh with a spacing of $0.008 D$, and the time step is 0.0005 . The maximum CFL number is approximately 0.08 , which is much lower than 1. A uniform free streamwise velocity $U_{\infty}$ is prescribed at the inlet boundary, and an average static pressure of zero is set across the outlet boundary $(p=0)$. The no-slip condition is prescribed at the cylinder surface, and the free-slip condition is imposed on the remaining domain boundaries. The Reynolds number is defined as $R e_{D}=U_{\infty} D / v$, where $U_{\infty}$ is the freestream velocity and $v$ is the kinematic viscosity. In this paper, the Reynolds number is set to 100 because the 3D effects can be neglected at this low Reynolds number (McClure and Yarusevych 2017).

Synthetic PIV data are obtained by linearly interpolating the simulated data in the region $1 D \leq x \leq 7 D$ and $-2.5 D \leq y \leq 2.5 D$, and the region is the same as the real measurement domain introduced in Sect. 4.1. After sampling, the spatial spacing and temporal step are $0.0502 D$ and 0.001 , respectively. This implies that the acquisition frequency is up to $1000 \mathrm{~Hz}$. The grid number of the data is $99 \times 119$ in the $x$ and $y$ directions, and 2368 frames are sampled in a time sequence. To more accurately simulate the real PIV data, correlated Gaussian noise is considered to assess the performance of the present method (Azijli and Dwight 2015; Andrea and Bernhard 2016). Gaussian random noise is added to the simulated velocity field as

$u_{n}=u \cdot\left(1+\varepsilon_{u} \cdot \Gamma_{u}\right)$

$v_{n}=v \cdot\left(1+\varepsilon_{u} \cdot \Gamma_{v}\right)$,

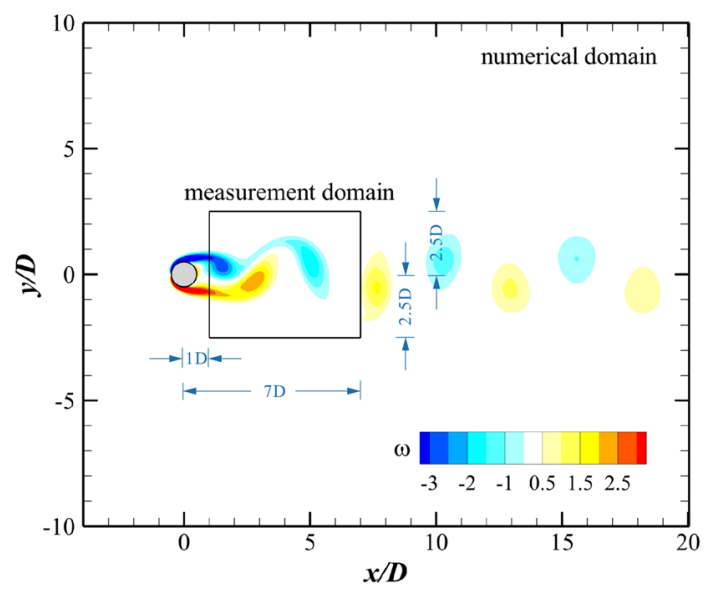

Fig. 3 The numerical domain of the simulation of the 2D flow around the circular cylinder. A PIV experiment at the same Reynolds number is also conducted in the region, as indicated by the measurement domain. The diameter of the cylinder is $10 \mathrm{~mm}$, and the Reynolds number based on that diameter is 100 where $u$ and $v$ are the velocity field data and $u_{n}$ and $v_{n}$ are the noisy data. Normally distributed random noise $\Gamma_{u}$ and $\Gamma_{v}$ with a standard deviation proportional to the magnitude of each velocity component is added to each exact value (John et al. 2010). The noise level $\left(\epsilon_{u}\right)$ is varied between 0 and $3 \%$ in $0.25 \%$ increments to estimate the error response. We do not consider the large-amplitude outliers because they are easily detected, removed and replaced by many methods (Westerweel and Scarano 2005; Garcia 2011; Wang et al. 2015). The spatially correlated Gaussian noise is obtained by averaging the independent noisy data with a $3 \times 3$ kernel (Wang et al. 2017). According to the paper by Garcia (2011), the error is estimated using the normalized root mean squared error (NRMSE) between the original $\left(\mathbf{U}^{0}\right)$ and optimized data $(\hat{\mathbf{U}})$ given by

$\mathrm{NRMSE}=0.5 \cdot\left(\frac{\left\|\hat{u}-u^{0}\right\|_{F}}{\left\|u^{0}\right\|_{F}}+\frac{\left\|\hat{v}-v^{0}\right\|_{F}}{\left\|v^{0}\right\|_{F}}\right) \times 100$.

Here, $\|\cdot\|_{F}$ denotes the Frobenius norm. Because the velocity will gradually converge to the real solution over time, the first 400 frames and the boundary points are ignored when calculating the NRMSE. The performance of PIV-PCS will be quantitatively compared with the median filtering, DCT-PLS and divergence-free smoothing (DFS) methods. The median filter is a nonlinear filtering method used to reduce noise, and it is commonly used when processing PIV data. DCT-PLS, proposed by Garcia (2011), combines a penalized least square approach and the discrete cosine transform to smooth the PIV data. DFS imposes a divergence corrective scheme on the PLS-DCT method and can smooth out both noise and divergence errors simultaneously (Wang et al. 2016a).

\subsection{About the parameter $s$}

Because of the measurement error, the weight coefficient $s$ is used to balance the results between the numerical solution and the measurements. The value of $s$ depends on the condition of Eq. 10. Figure 4 shows the 2D contours of the dependency of the NRMSE of the velocity on the noise level under different $s$. The BCs are addressed without and with POD in Fig. 4a, b, respectively. Because the error monotonically decreases with increasing $s$ from 0 to 0.94 , we only consider the parameter $s$ in the range from 0.94 to 1.0 with an increment of 0.005 . Overall, the error is very small. It is apparent that the error increases with the noise level for a fixed $s$. Moreover, the error exhibits a rapid increase when $s$ gradually approaches 1 for both BCs-Raw and BCs-POD. This implies that incorrect values of the BCs will reduce the fidelity of the numerical simulations. According to the contour map, we propose an optimal $s$ of 0.985 for this case. Substituting the optimal value into Eq. $10, \alpha$ should be on the 


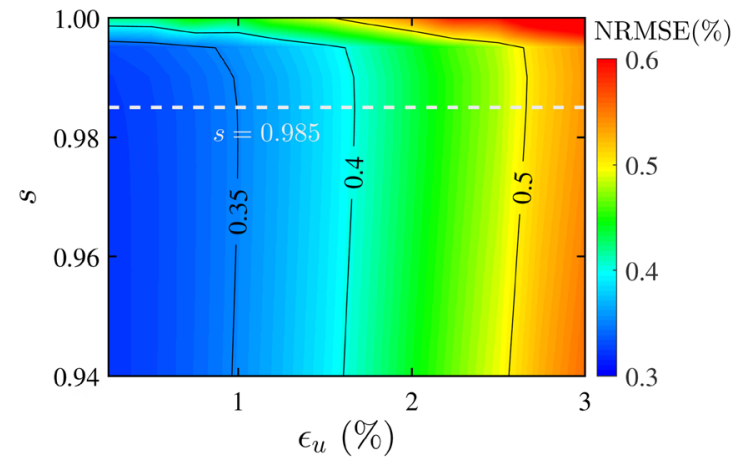

(a)

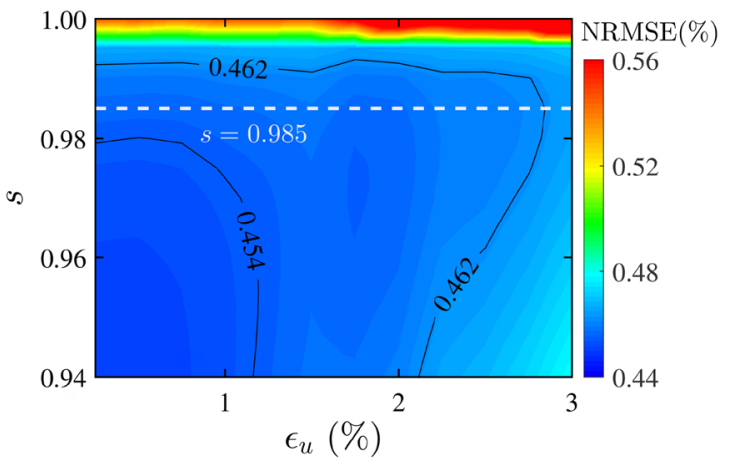

(b)

Fig. 4 The 2D contour map of the NRMSE as a function of noise level $\left(\epsilon_{u}\right)$ and $s$. The parameter $s$ is only presented in the range of $0.94 \leq s \leq 1.0$. a BCs-Raw, b BCs-POD. Note that the range of the colormap is different

order of 10 . This is why we suggest that $\alpha$ is approximately 10 in Sect. 2.2 for the PIV-PCS method.

We also plot the root mean square (RMS) of the difference between the numerical simulation and PIV-PCS as a function of time in Fig. 5. The left and right planes are for the streamwise and vertical velocities, respectively. The RMS difference of PIV-PCS with $s=0.985(\alpha \approx 10)$ is less than those of $s=0.5$ and 1.0. For the streamwise velocity, the errors in the PIV-PCS are approximately constant over time. In contrast, the error given by $s=1.0$ for the vertical velocity presents an increasing trend over time due to the artificial noise. A proper $s(s=0.985)$ can suppress the increase in instabilities. In addition, the error convergence rate of $s=0.985$ is much faster than that of $s=1.0$.

If the sampling frequency is reduced to $50 \mathrm{~Hz}$ (still far greater than the vortex shedding frequency), the choice of $\alpha$ remains suitable. In this case, the figures, which are similar to Figs. 4 and 5, are provided in the "Appendix". According to Eq. 10, the optimal $s$ is approximately 0.7 .

\subsection{The performance with respect to reducing error}

First, the performance of the PIV-PCS method is evaluated without artificial noise. The exact boundary conditions are obtained without applying smoothing by POD. Figure 6 shows the instantaneous contour maps of the vorticity $(\omega)$ and normalized pressure $\left(\frac{p}{1 / 2 \rho U_{\infty}^{2}}\right)$ for the original data and the present method, respectively. Both the vorticity fields and pressure fields calculated by the present method are essentially identical to the original fields. The NRMSEs of the velocity, vorticity and pressure are approximately $0.4 \%$, $1.4 \%$ and $0.3 \%$, respectively. This means that the velocity can be recovered with high accuracy by the present method without considering noise.

The performance of the PIV-PCS method is assessed on the synthetic PIV data with spatially correlated noise, and compared with the DCT-PLS, median filter and DFS methods in terms of PIV error reduction. Figure 7 shows instantaneous vorticity contours addressed using different methods. The original data with spatially correlated noise are displayed in Fig. 7a, and the data processed by the $3 \times 3$ median filtering, DCT-PLS with robust smoothing, DFS and the present PIV-PCS method with $s=0.985$ are presented in Fig. 7b-e, respectively. The noise level is $2 \%$. From Fig. 7b, c, we can see that the DCT-PLS and median filter methods cannot smooth out the correlated noise. Note that conventional filtering methods will also smoothen the
Fig. 5 The time evolution of the RMS difference between numerical simulation and PIVPCS for the streamwise velocity (a) and vertical velocity (b). The black, purple, green and red lines represent the results with artificial noise and PIV-PCS, with $s=0.5,0.985$ and 1.0 . The noise level $\epsilon_{u}$ is $5 \%$. The range of the y-coordinate is different

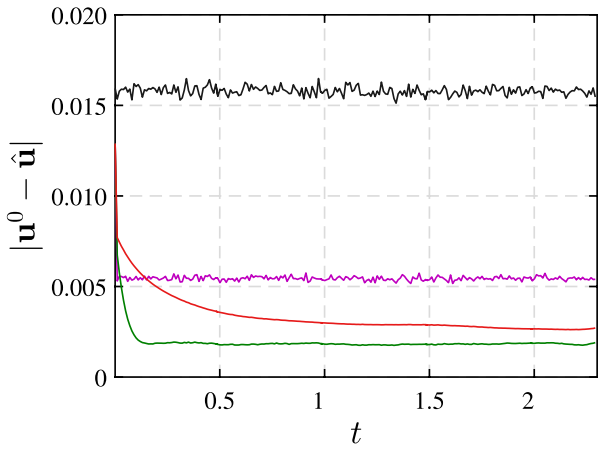

(a)

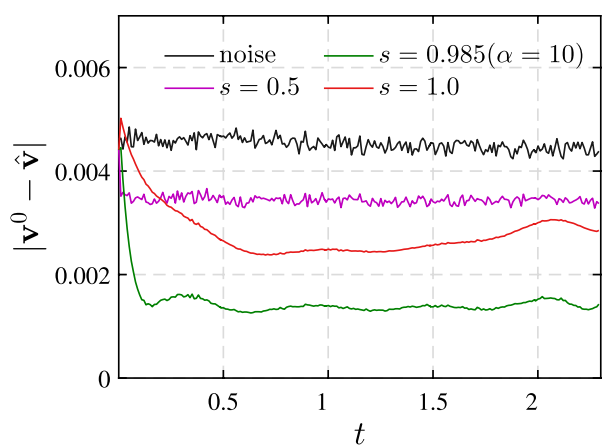

(b) 
Fig. 6 A comparison between the original vorticity and pressure field with those calculated from the present numerical method without adding noise. a, b An instantaneous vorticity field. c, d An instantaneous pressure field. The left and right planes are for the original data and the present method, respectively

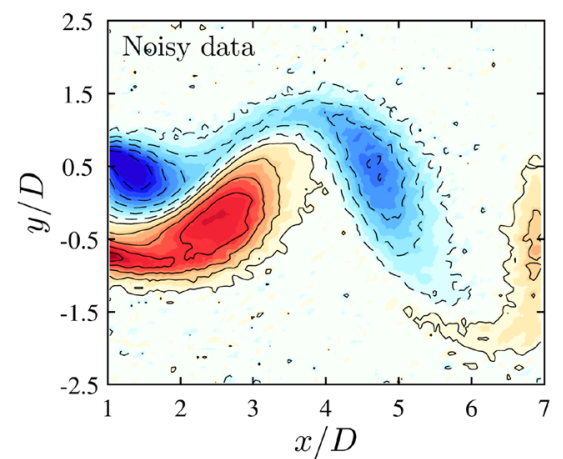

(a)
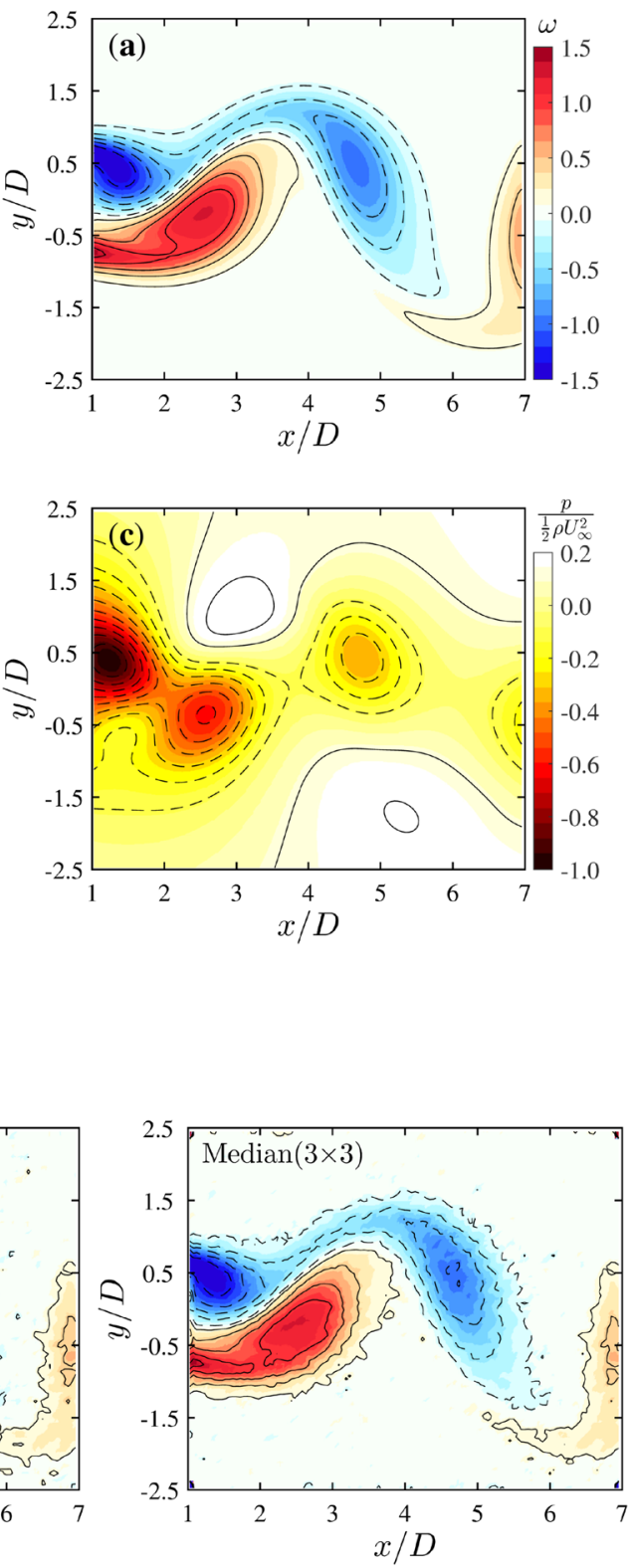

(b)
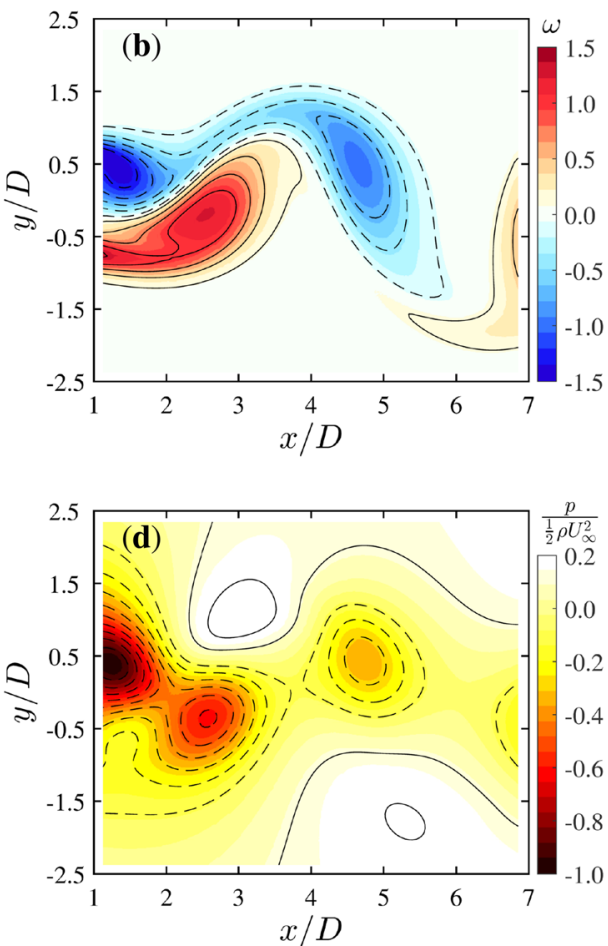

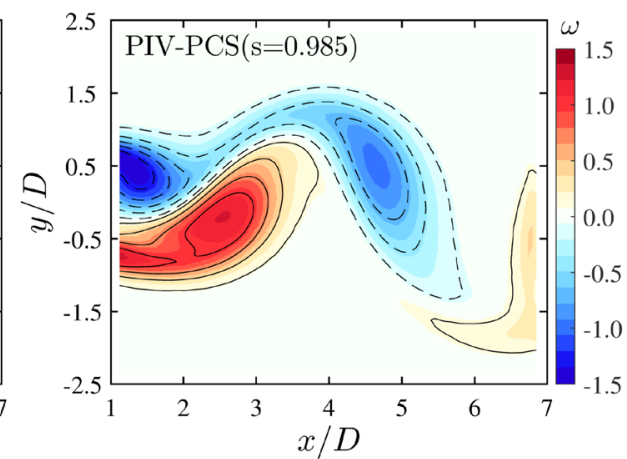

(e)

ticity field processed by the robust DCT-PLS; $\mathbf{d}$ The vorticity field processed by DFS; e The vorticity field processed by the present PIV-PCS method
Fig. 7 An instantaneous vorticity field. a Raw vorticity field with spatially correlated noise, where the noise level is $2 \%$. b The vorticity field processed by median filtering with a size of $3 \times 3$; $\mathbf{c}$ The vor- (c)

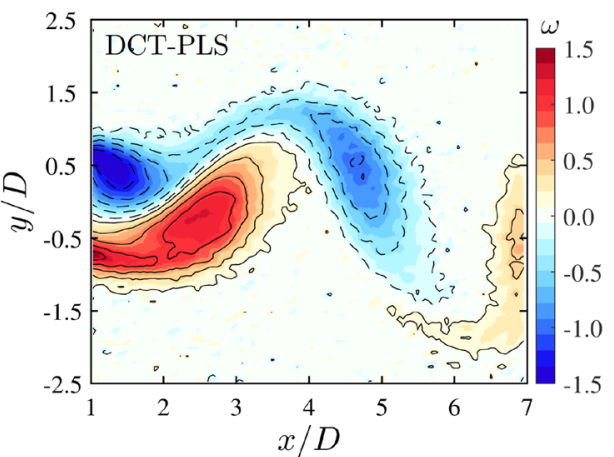

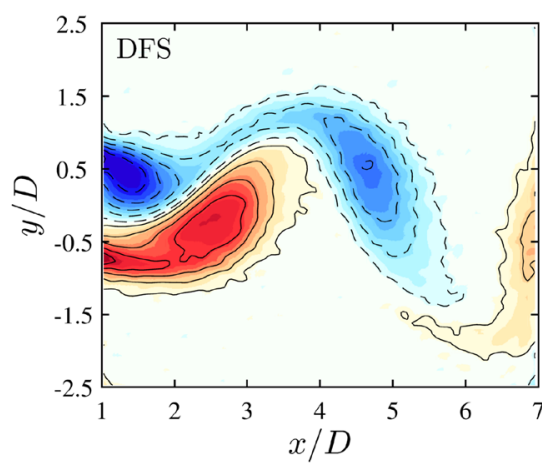

(d) 
original correct data. The DFS achieves a better performance using the divergence-free constraint, although the contour lines remain distorted by unexpected noise. From visual inspection of the contours, the presented method shows the best performance on reducing the correlated noise among all the tested methods.

The quantitative comparison with respect to error reduction is given in the plots of Fig. 8. The NRMSEs of the velocity and vorticity are given in Fig. 8a, b, respectively. Note that the range of the vorticity NRMSE is much larger than that of the velocity. When the velocity field is free of noise, none of the methods can fully recover the velocity because the velocity is already smoothened by the average kernel with size of $3 \times 3$ when adding the correlated noise. This result is proved in Fig. 8c, d, where the NRMSEs of the velocity and vorticity are plotted as a function of filter size under the free-noise condition. The errors in both the velocity and vorticity increase with increasing filter size. For the velocity, the error in the PIV-PCS is always slightly larger than the spatial filtering because of the incorrect boundary conditions. For the vorticity, the error in the present method is higher than average filtering when the filter size smaller than 3 and slightly lower than that of average filtering with increasing filter size. The PIV-PCS can not reduce the modulation error of PIV data, this implies that the PTV data with high resolution is more suitable for the present method. Another phenomenon observed in Fig. 8a is that the velocity is deteriorated by the boundary filtering when the noise level $\epsilon_{u}$ is less than $2 \%$, while the error in the vorticity is almost the same within the whole range of noise levels. Overall, the error in PIV-PCS presents a slowest-growth trend with increasing noise level among all the methods in terms of velocity and vorticity.

\subsection{The performance in terms of estimating pressure}

From the discussions in Sect. 3.2, we know that the measurement error can be reduced by the present PIV-PCS method. However, the influence of error on pressure is more complicated than that on velocity. The pressure solved from Eq. 6 is not what we expect because the term on the right-hand side, $\nabla \cdot \mathbf{U}^{* *} / d t$, is sensitive to the noise. Mathematically, the pressure solved from Eq. 6 is used to make the velocity satisfy the divergence-free condition. As analyzed in the PIV-based pressure measurements, the error will propagate from the velocity field to the pressure field (de Kat and van Oudheusden 2012; Pan et al. 2016). de Kat and van Oudheusden (2012) proposed that the RMS error of the pressure $\delta_{p}$ for Eulerian form can be given as
Fig. 8 The NRMSEs of the velocity (left plane) and vorticity (right plane) as a function of noise level $\epsilon_{u}(\mathbf{a}, \mathbf{b})$ and average filter size $(\mathbf{c}, \mathbf{d})$ without synthetic noise. In figure (a, b), the PIV-PCS with BCs-Raw $-\circ$ and $\mathrm{BCs}-\mathrm{POD} \_$- are compared with median filtering $\rightarrow$, DCT-PLS $\multimap-$ and DFS

$\rightarrow$. In figure $(\mathbf{c}, \mathbf{d})$, the errors caused by the present method with BCs-Raw - - - and average spatial filter with different sizes $\leftrightarrow$ are presented

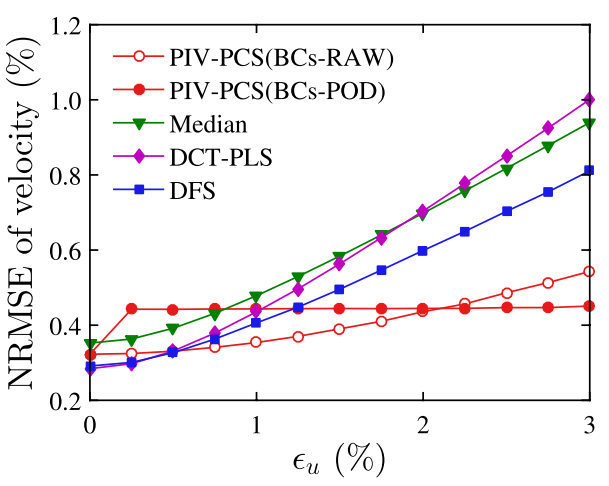

(a)

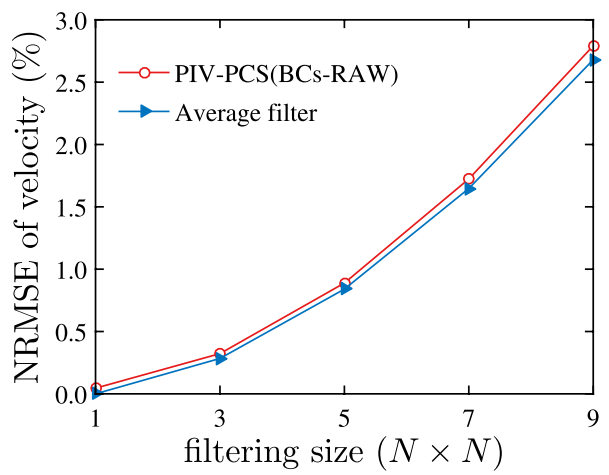

(c)

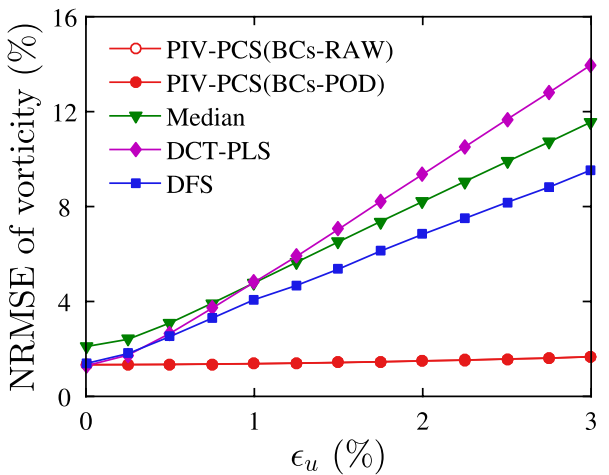

(b)

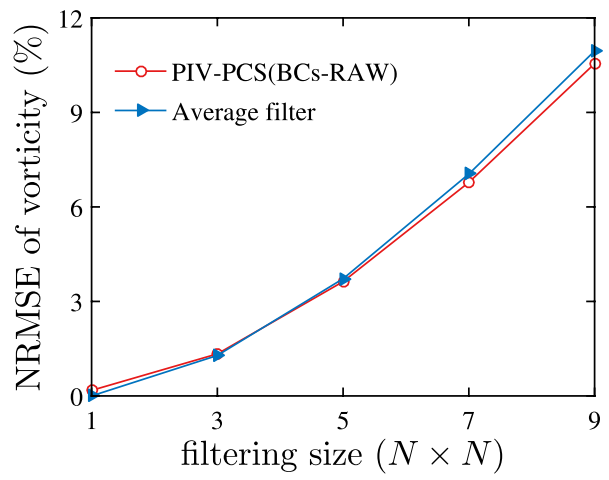

(d) 
$\delta_{p} \propto \delta_{u} \sqrt{\frac{h^{2}}{2 d t^{2}}+|\nabla u|^{2} h^{2}+\frac{|\mathbf{U}|^{2}}{2}}$,

where $\delta_{u}$ is the noise in the velocity, $h$ is the grid spacing, $d t$ is the velocity time separation, $|\nabla u|$ is the magnitude of the gradient of the streamwise component of the velocity, and $|\mathbf{U}|$ is the velocity magnitude. For TR-PIV, the error can be amplified by a small time separation, and the error in the pressure is dominated by the first term of the right-hand side, that is,

$\delta_{p} \propto \delta_{u} \sqrt{\frac{|\mathbf{U}|_{\max }^{2}}{2 \cdot C F L^{2}}}$.

Here, $|\mathbf{U}|_{\max }$ is the maximum of the velocity magnitude, and the CFL number is defined as Eq. 11. This result indicates that the error in the pressure increases with decreasing CFL number, although a small CFL number is beneficial to the stability of the numerical solution.

In this context, the CFL number of the synthetic PIV data is approximately 0.026 . If the boundary velocities are not smoothened by any filtering approaches, the error in the initial pressure estimated from the noisy PIV data is approximately 27 times higher than that of the velocity, and PIV-PCS cannot decrease the error in the pressure. Therefore, a method of POD-based smoothing is adopted to minimize the effects of measurement noise at the boundaries.

The NRMSEs of the pressure obtained by the present method are compared with the result from directly solving the Poisson equation (Oudheusden 2013), where the pressure is discretized using a standard five-point formula and the gradient of the velocity is estimated by a second-order central-difference scheme. The boundary velocity is also filtered using the POD method. The NRMSEs of the pressure are plotted in Fig. 9a, where the filled regions indicate the standard deviation of the pressure field error. The pressure error of the present method increases from 0 to $23 \%$ as the noise levels increase, and that of the pressure Poisson approach increases from 4 to $10 \%$ across the whole range of noise levels. The NRMSEs of the pressure under the present method exhibit a stronger noise sensitivity than directly solving the pressure Poisson equation. The error in the Poisson approach is up to $4 \%$ at $\epsilon_{u}=0 \%$. To explore the reason for this, we plot the NRMSE of the pressure as a function of filter size (Fig. 9b); there is no noise in the synthetic PIV data. In this figure, the pressure error in the present method is substantially smaller than that of the Eulerian approach. This figure indicates that spatial smoothing can contaminate the pressure solution, especially when directly solving the pressure using Poisson's equation.

An advantage of the PIV-PCS method is that the pressure can be directly solved again from the optimal velocity fields. The boundary conditions are imposed from the PIV data; thus, POD-based smoothing must still be applied when solving the Poisson equation. The error in the pressure for each $\epsilon_{u}$ is shown in Fig. 9c and compared with that of DFS. First, comparing Fig. 9a, c, it is obvious that the divergencefree correction can improve the accuracy of the pressure estimation, although the improvement is not very significant. The error in the pressure for DFS still increases from 0 to $10 \%$ when the noise level increases from 0 to $3 \%$. Second, the pressure error, solved from the velocity field of PIV-PCS, is reduced to approximately $2.2 \%$ across the whole range of noise levels, being much lower than that of DFS. This

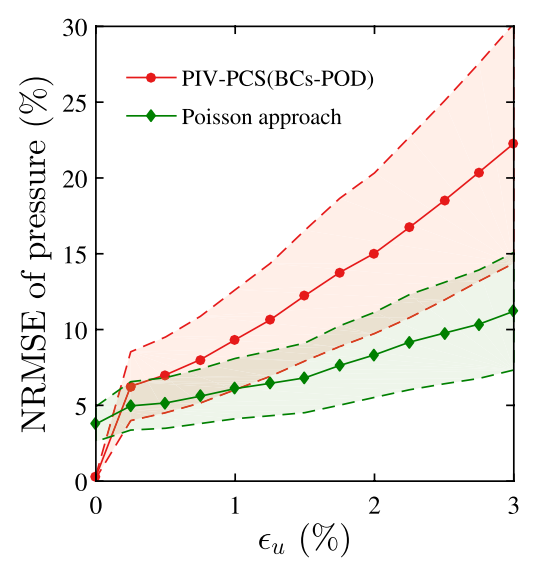

(a)

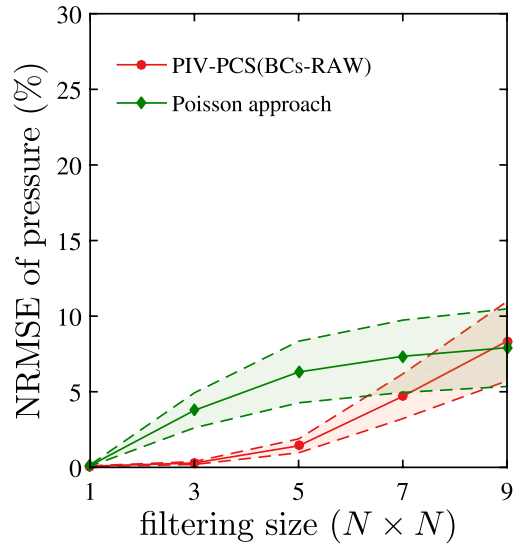

(b)

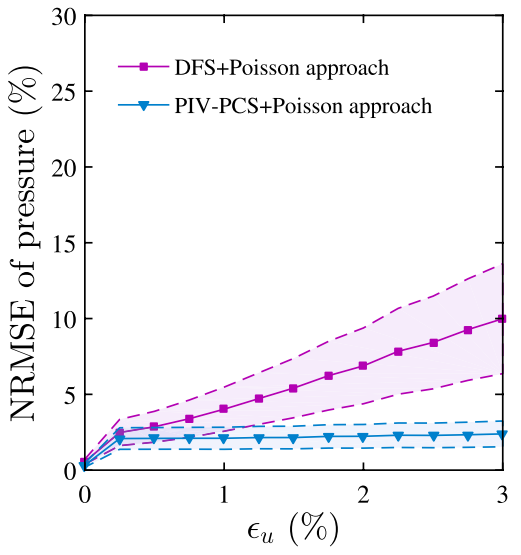

(c)
Fig. 9 The NRMSEs of pressure, where filled regions indicate the standard deviation of the pressure field error. a NRMSEs of pressure as a function of noise level, where the red represents the present method and the green represents the result from directly solving the pressure Poisson equation (Poisson approach). b The NRMSEs of the pressure as a function of filtering size without noise. No special process is applied to the boundary. c The pressure field is directly solved from the velocity field obtained from the DFS and PIV-PCS methods 
implies that the present method can provide an optimal velocity field for solving the pressure.

\section{Experimental applications}

To verify that the PIV-PCS proposed in this paper is also suitable for real-world experiments, two PIV data sets are studied. The first data set is a 2D wake flow around a circular cylinder at the same Reynolds number with the previous numerical simulation in Sect. 3. The second data set is a $3 \mathrm{D}$ wake flow of a hemisphere at higher Reynolds number. Because there are no perfect reference solutions for experiments, we will use the velocity divergence and pressure-gradient curl to make a quantitative comparison. The boundary velocities are processed by BCs-POD. The results of DFS are also shown in this part for comparison. Especially, a temporal polynomial filtering (TF) is adopted within the first $2 \mathrm{D}$ case to inspect the differences between spatial filtering and temporal filtering.

\subsection{D wake flow of circular cylinder}

This experiment is conducted in a small, low-speed water channel with a work section of $380 \mathrm{~mm} \times 380 \mathrm{~mm} \times 800$ $\mathrm{mm}$ (height $\times$ width $\times$ length). To compare with the numerical solution, the Reynolds number based on the cylinder diameter is also set to 100 . The free-stream velocity $U_{\infty}$ is 10 $\mathrm{mm} / \mathrm{s}$, with the free-stream turbulence intensity $T u$ being less than $0.6 \%$. Note that $T u$ is calibrated by PIV measurement in the case of free streaming; therefore, the free-stream turbulence intensity may be overestimated due to the noise in the PIV data. An acrylic-glass circular cylinder with a diameter of $10 \mathrm{~mm}$ was horizontally positioned in the middle plane of the water channel, and the length of the cylinder is $380 \mathrm{~mm}$. The condition of the 2D flow is guaranteed in terms of the high aspect ratio of the cylinder and low Reynolds number.

The flow field is measured by a 2D TR-PIV system, which contains a semiconductor laser generator and a high-speed PHOTRON camera. The continuous 532-nm laser beam was expanded to a laser sheet with a thickness of approximately $1.0 \mathrm{~mm}$, and the output power is approximately $4.0 \mathrm{~W}$. The plane of the laser is located along the middle of the cylinder in the axis direction. The particle images are recorded by a high-speed CMOS camera (Fastcam SA8/15K-M2) with a resolution of $1280 \times 1024$ pixels at a sampling frequency of $500 \mathrm{~Hz}$. The long side of the image is along the streamwise direction. A 50-mm, f1.4 SIGMA prime lens is used. This configuration yields a digital resolution of approximately 17 pixels $/ \mathrm{mm}$. The tracers are hollow glass micro-spheres with a mean diameter of $12 \mu \mathrm{m}$, and the density is $1.1 \mathrm{~g} / \mathrm{cm}^{3}$. The images are captured $10 \mathrm{~min}$ after seeding the particles so that the tracers would be uniformly distributed in the water.
Limited by the memory of the camera, 4000 images are captured in one run within approximately $8.0 \mathrm{~s}$.

Before calculating the velocity, image processing, including background subtraction and Gaussian smoothing, is applied to the raw particle images to minimize the noise. Because of the small displacement between two neighboring frames, the correlation function is computed from two records at a separation of 18 frames, corresponding to a maximal displacement of approximately 10 pixels. Image analysis is performed with a window deformation iterative multigrid technique [WIDIM, Scarano (2002)], where the final window size is $32 \times 32$ pixels with $75 \%$ overlap. The measured velocity field is smoothened to be free of obvious outliers (Westerweel and Scarano 2005). The velocity fields are cropped corresponding to the measurement domain indicated in Fig. 3. This yields a measurement grid of $126 \times 105$ with a spacing of $0.048 \mathrm{D}$. According to Eq. 10, the optimal $s$ is approximately 0.975 .

The instantaneous field of DNS, raw PIV data, DFS, TF and PIV-PCS (from top to bottom) are shown in Fig. 10. To display the same flow structure, the DNS data were chosen by finding the maximum correlation coefficient between the DNS fields and the PIV fields. 21-point kernels were used in temporal polynomial filtering. The vortical structure is identified using the $\lambda_{\mathrm{ci}}$ method. The value of $\lambda_{\mathrm{ci}}$ is defined as the imaginary part of the eigenvalue of the velocity gradients (Zhou et al. 1999), and the rotational direction is given by the sign of the vorticity. The $\lambda_{\mathrm{ci}}$ field of the raw PIV data is distorted by the noise, and the center of the vortices is difficult to be located. Although the DFS method improves the identification of vortical structures, the pressure contour of DFS is almost the same as the raw PIV data. After being processed by PIV-PCS, the $\lambda_{\mathrm{ci}}$ field is very smooth, and the shape of the vortices can be clearly defined. The pressure distribution of PIV-PCS and TF are closest to that of DNS from visual comparison. Note that the pressure in Fig. $10 \mathrm{j}$ is calculated by solving the Poisson equation from the PIV-PCS velocity fields. The magnitudes of both the vorticity and pressure are smaller than those of DNS; this is caused by the spatial low-pass filtering introduced by the PIV interrogation.

The time series of the RMS of the divergence, RMS of the curl of the pressure gradient, the vertical velocity $v$ and the pressure $p$ are presented in Fig. 11. The divergence is estimated by employing a central discretization in the interior grids and a one-side differencing scheme at the boundary points. The pressure gradient is estimated from the velocity fields by employing the $\mathrm{N}-\mathrm{S}$ equations. The standard deviation of the divergence and curl for every time step is plotted in Fig. 11a, b. Both DFS and PIV-PCS can reduce the divergence to a low level; however, the PIV-PCS provides a smaller curl, approximately 1, than DFS, approximately 7.5. The TF method can significantly reduce the pressure 
Fig. 10 Instantaneous fields at $t=2.0$. The left plane displays the contours of the vortical structure, and the right plane displays the contour maps of the pressure. From top to bottom: the results from DNS, the experimental data, DFS, temporal polynomial filtering with 21-point kernels and PIV-PCS with $s=0.975$. The absolute contour levels represent $\lambda_{\mathrm{ci}}$ from 0.1 to 1.0 in steps of 0.2 . The positive contour levels of the pressure are from 0.1 to 0.2 in steps of 0.1 , and the negative levels of the pressure are from -1.1 to -0.1 in steps of 0.1
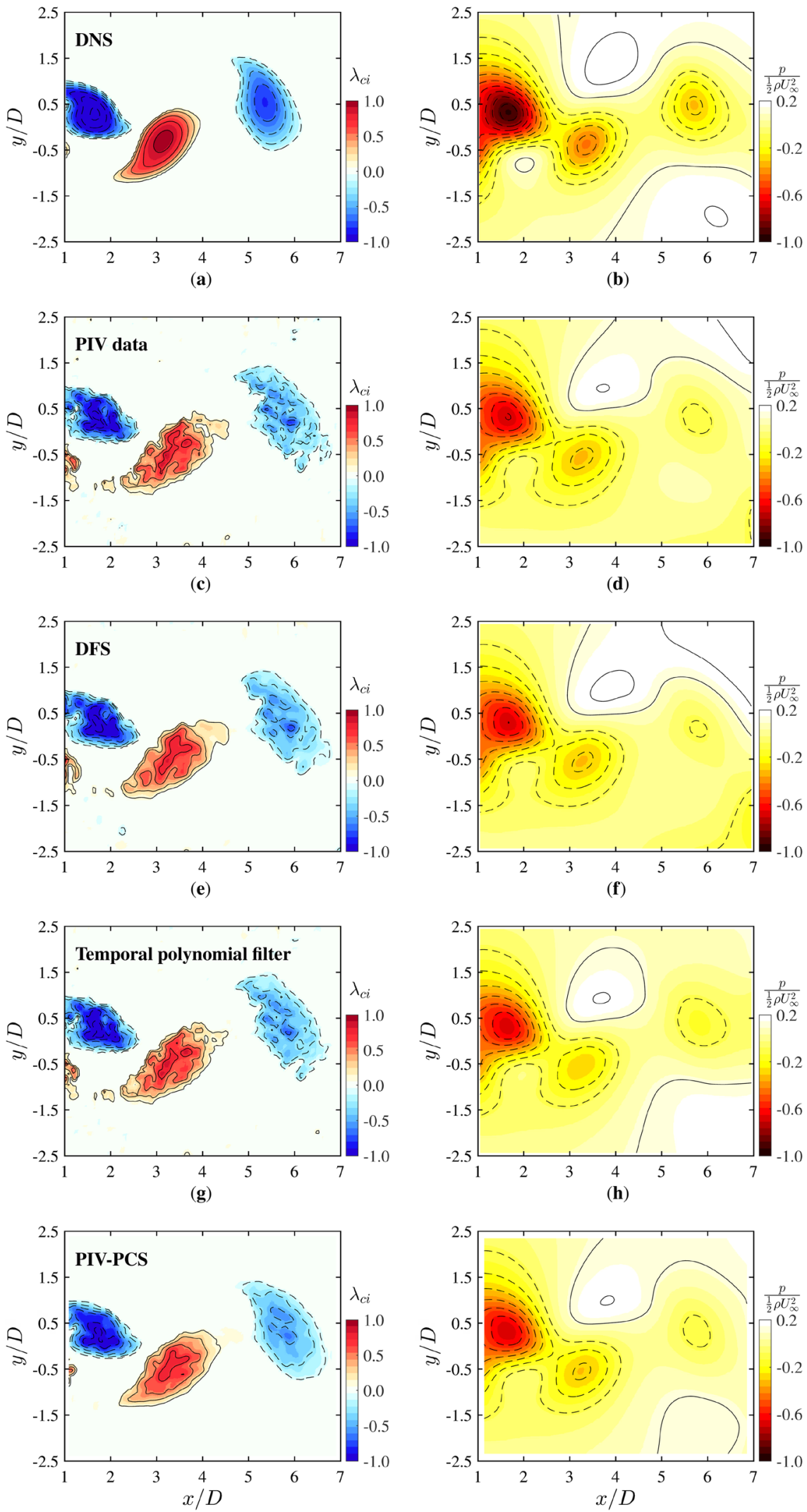

(i)

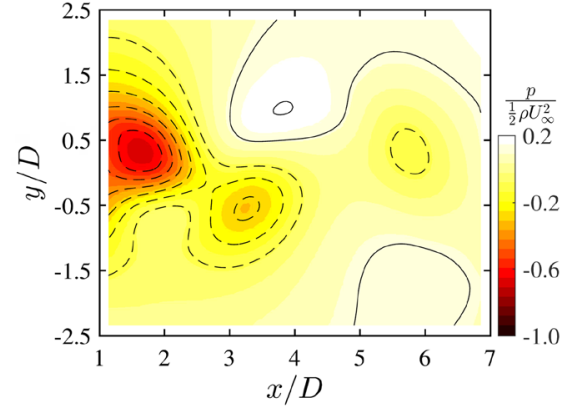

(j) 
curl, though it has little influence on velocity divergence. The vertical velocity at the center of the measurement domain $(x / D=4, y / D=0)$ is shown in Fig. 11c. For comparison, the velocity signal in the time range of $[4,5]$ is zoomed in, as shown in the sub-figure. We can see that the velocity fluctuations of the raw PIV data, DFS and TF are much higher than those of PIV-PCS. These non-physical velocity fluctuations can result in a failure in calculating time derivatives. The red curves obtained by PIV-PCS are very smooth in the time direction and exhibit a good consistency with the experimental data. In Fig. 11 d, the pressure shows a similar behavior as the vertical velocity at the same point. The pressure obtained by DFS is almost the same as that from the raw PIV data, and the TF and PIV-PCS can result in more accurate pressure. This implies that only using a constraint on the spatial divergence is not sufficient to recover the real velocity fields.

\subsection{D wake flow of hemisphere}

This tomographic experiment on the 3D wake flow of a hemisphere was designed to investigate the three-dimensional formation and generation of hairpin vortices in a laminar boundary layer. The hairpin vortex is one of the principle characteristic elements in wall-bounded turbulence (Smits et al. 2011), and it plays a key role in coherent structures, as described in Adrian et al. (2000) and Adrian (2007). Acarlar and Smith (1987a, b) studied the hairpin vortex generated by the interaction of a hemisphere or fluid injection with a developing laminar boundary layer. Both flow visualization and anemometry measurements were applied to examine the characteristics of the hairpin vortex. In this context, we will not focus on the physical process of the development of the hairpin vortex; our goal is to optimize the tomographic PIV data using the PIV-PCS method. An investigation of the physical flows can be found in Wang (2017), which will be published as a journal article later.

This experiment was performed in a water tunnel at Beihang University, China. A hemisphere with a diameter $D$ $=20 \mathrm{~mm}$ was mounted on a flat plexiglass plate, and the plate was vertically posited in the test section of the water tunnel. The plate is $2 \times 0.5 \mathrm{~m}^{2}$, and an elliptic leading edge was employed. The hemisphere was located at $0.7 \mathrm{~m}$ downstream from the leading edge to guarantee the condition of a laminar boundary layer. The velocity profile of the boundary layer was calibrated using laser Doppler velocimetry (LDV). The data used in this paper were gathered at a free-stream velocity of $145 \mathrm{~mm} / \mathrm{s}$. Therefore, the Reynolds number based on the hemisphere diameter $R e_{D}=V_{\infty} D / v$ is approximately 2750, where $v$ is the kinematic viscosity of water at a temperature of $18{ }^{\circ} \mathrm{C}$. The $x$ direction is defined as in the direction of the free-stream velocity, and the $z$ direction is defined as the wall-normal direction.

A TR-tomographic PIV system was used to record the images and quantitatively analyze the velocity fields. The
Fig. 11 Time histories of divergence (a), curl of pressure gradient $(\mathbf{b})$, vertical velocity (c) and pressure (d). The divergence and curl are estimated as the standard deviation at every time step, and the vertical velocity and pressure are estimated at the center point of the measurement domain. For comparison, the results of DFS and TF (temporal polynomial filtering) are also plotted

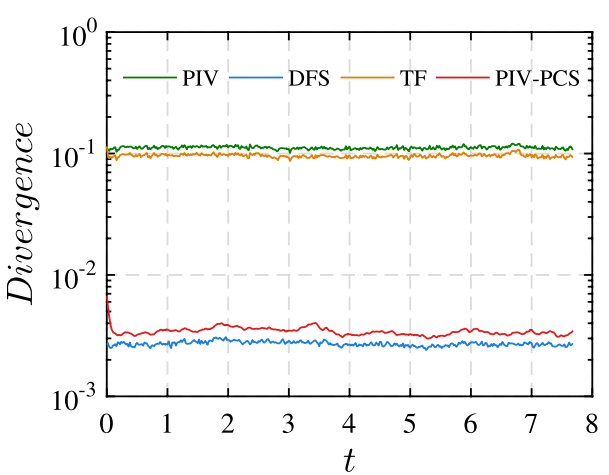

(a)

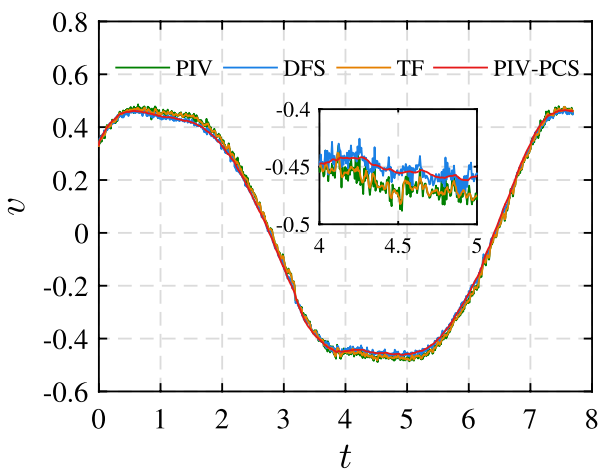

(c)

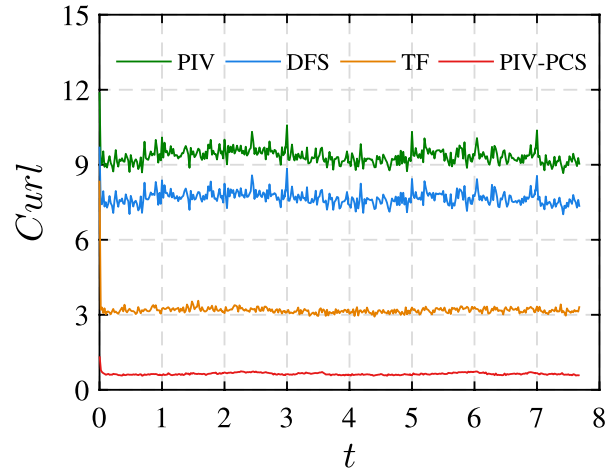

(b)

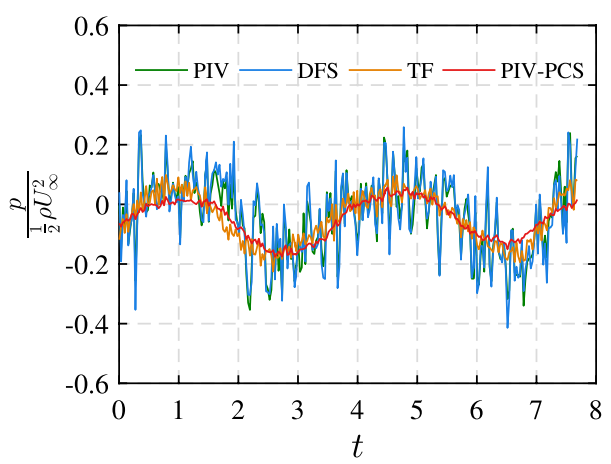

(d) 


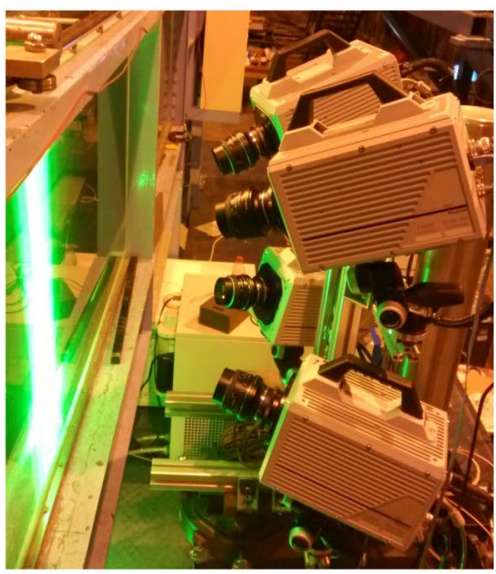

(a)
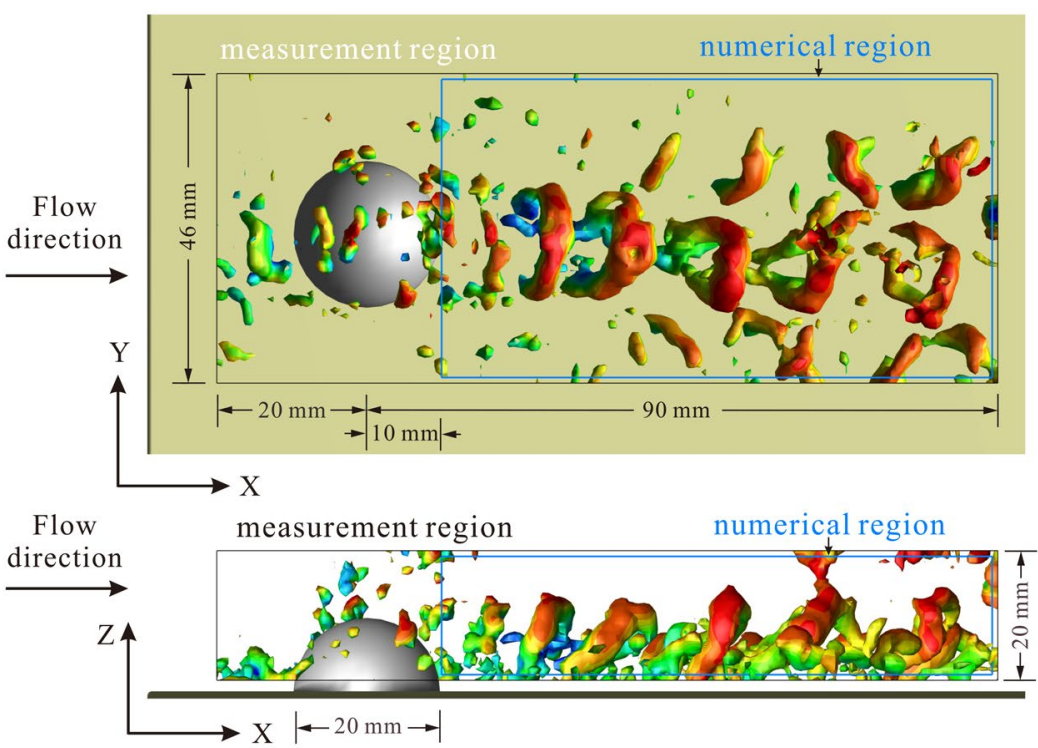

(b)
Fig. 12 a Experimental set-up for tomographic PIV; b the schematic top view (top) and side view (bottom) of the flow structures. These vortex structures are identified with $\lambda_{\mathrm{ci}}=1.3$ and colored based on

experimental set-up of the tomographic PIV is shown in Fig. 12. A high-frequency double-pulse laser with a wavelength of $532 \mathrm{~nm}$ was used to illuminate the measurement volume; the energy of the laser was set to $30 \mathrm{~mJ} / \mathrm{pulse}$. The thickness of the illuminated volume is approximately $20 \mathrm{~mm}$, and the bottom of the volume approaches the wall. Hollow glass beads with a median diameter of $10 \mu \mathrm{m}$ and density of $1.05 \mathrm{~g} / \mathrm{cm}^{-3}$ were used to seed the flow field, and their motions were captured by four Photron high-speed CMOS cameras (Fastcam SA2), which were laterally placed to form a ' $X$ ' shape. The view angle between any two cameras was approximately $30^{\circ}$. The image resolution was set to $2048 \times$ 1024 pixels to fit the measurement region and reduce the camera memory requirements. The digital resolution was approximately 16 pixels $/ \mathrm{mm}$, and the particles per pixel (ppp) was approximately 0.05 .

The particle images were recorded at the frequency of $250 \mathrm{~Hz}$, and the number of samples was 1250 . Before reconstructing the intensity volume, the raw images were processed by subtracting the background, performing a sliding minimum and smoothing with a Gaussian filter. The measured volume, with a size of $110 \mathrm{~mm} \times 46 \mathrm{~mm}$ $\times 20 \mathrm{~mm}$, was reconstructed using the intensity-enhanced MART (Wang et al. 2016b). A volume deformation iterative multi-grid technique with a final interrogation volume size of $48 \times 48 \times 48$ at $75 \%$ overlap was used to estimate the displacement. The velocity fields were validated using normalized median test and smoothed using a Gaussian filter. The vector spacing is $0.768 \mathrm{~mm}=0.0384 \mathrm{D}$ along all three directions. the streamwise velocity. The blue box indicates the numerical region used by PIV-PCS

Because of the large time separation, the CFL number based on the free-stream velocity is approximately 0.8 , which is very high for numerical simulations. As introduced in Sect. 2.2, the large time separation will increase the truncation error and the instability of the numerical solution. The acquisition frequency $(250 \mathrm{~Hz})$ is slightly low to fully capture the time evolution of all the structures. However, it is sufficient for the main flow structures $(\sim O(10) \mathrm{Hz})$. According to Eq. 10, the optimal $s$ is 0.42 under this experimental configuration.

The PIV-PCS method is compared with DFS. Figure 13 shows an example of the instantaneous flow organization identified using the $\lambda_{\mathrm{ci}}$ method. The isosurfaces of $\lambda_{\mathrm{ci}}=0.6$ are colored based on the value of the streamwise velocity $u$. Figure 13a-d presents the results of the raw tomographic PIV data, DFS, and PIV-PCS with $s=0.42$ and 0.9. All the results clearly exhibit the primary hairpin vortex behind the hemisphere. These vortices have strong vorticity and periodically shed from the shear layer of the hemisphere. In addition to the primary flow structures, secondary hairpins are formed by the interactions between the lifted-up lowmomentum fluid and higher speed outer flows (Acarlar and Smith 1987a). The secondary hairpins indicated by the letters ' $L$ ' and ' $R$ ', representing left and right, respectively, cannot be clearly identified from the raw data. The secondary vortex structures are very clear after processing using the DFS and PIV-PCS methods, especially for those vortices upstream of the measurement region. In the original experimental data, a vortex at the location indicated with an arrow is not connected as a complete hairpin structure 


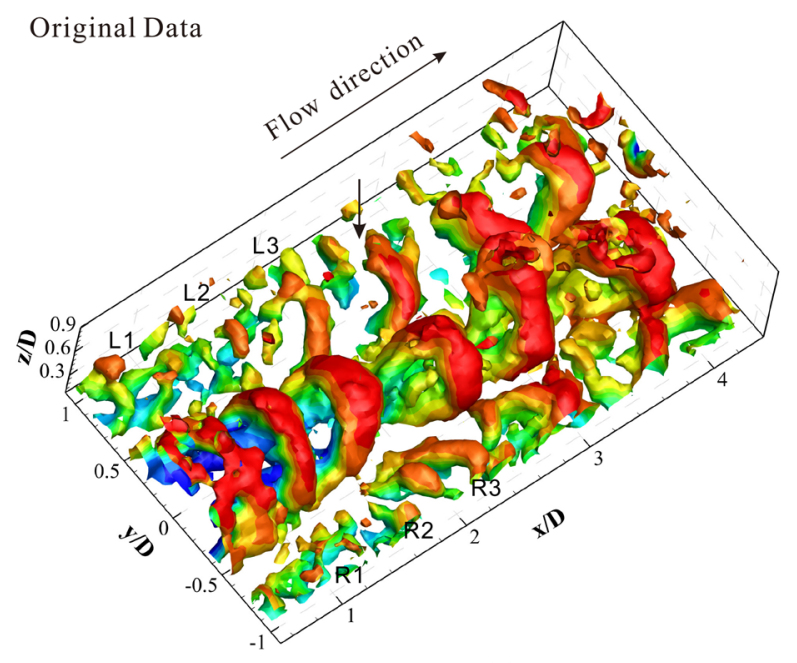

(a)

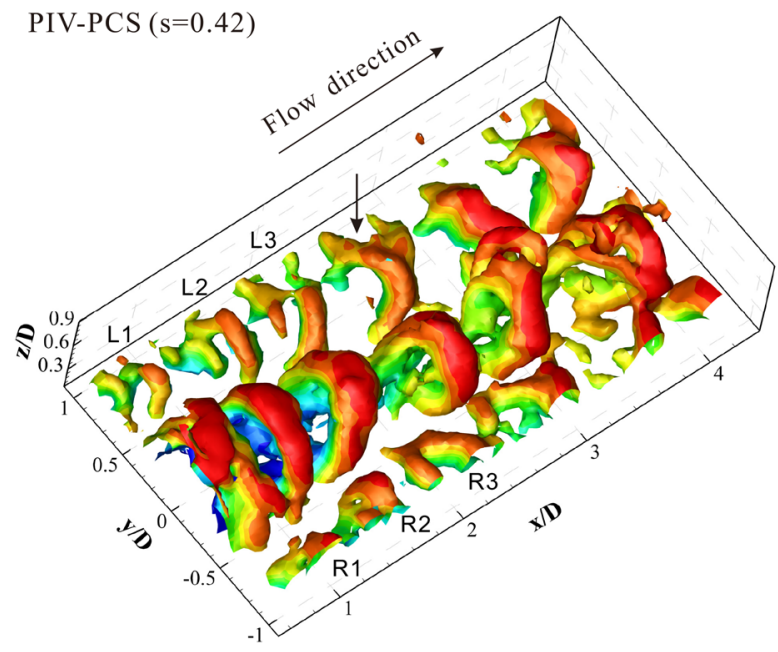

(c)

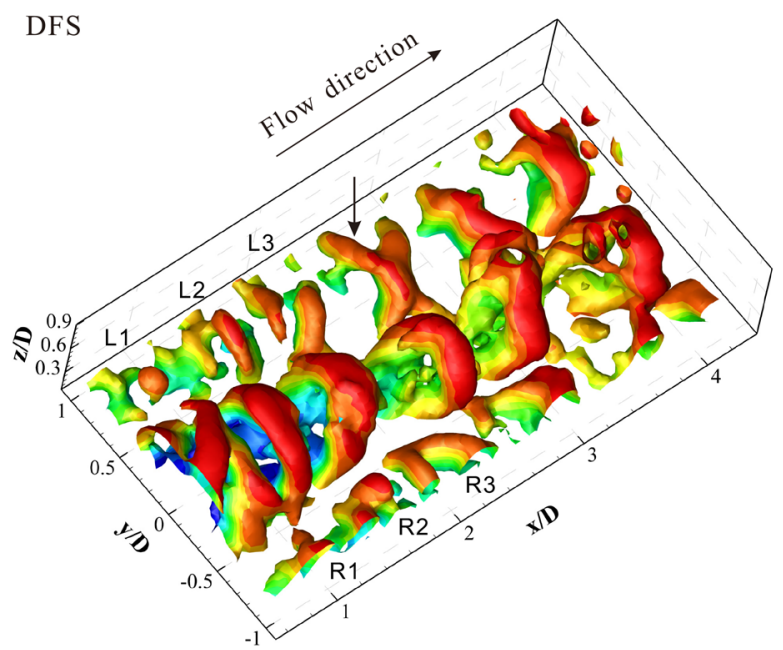

(b)

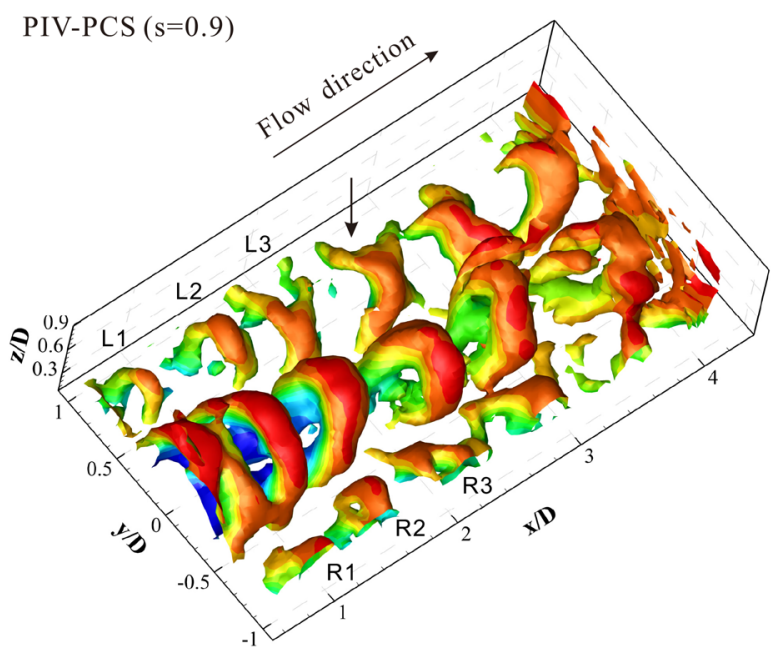

(d)

$\mathrm{U}$

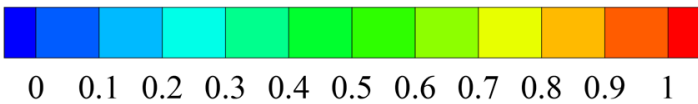

Fig. 13 Instantaneous flow field visualized by isosurfaces of $\lambda_{\mathrm{ci}}=0.6$. These flow structures are colored based on the streamwise velocity. The original velocity field (a) is processed by DFS (b) and PIV-PCS with $s=0.42$ (c) and 0.9 (d), respectively

due to the strong velocity fluctuations. However, after processing by DFS and PIV-PCS, an integral hairpin vortex is presented in the same location instead of a broken vortex. From visual inspection of the results, we can see that the results obtained using DFS and PIV-PCS with $s=0.42$ are very similar. The obvious difference appears at the location indicated by L1. The flow structure of PIV-PCS presents a better arch-shaped vortex compared to the structure of DFS. The measurement error is suppressed, and the flow structures are more distinct for both DFS and PIV-PCS. It is difficult to conclude which one is better from visual comparison alone. Thus, some quantitative comparisons will be given in the next paragraph. Note that an unphysical error is generated at the downstream edge of the measurement region when $s=0.9$. This is caused by the large separation time, which can increase the numerical instability and truncation error.

The probability density distributions (PDFs) of the divergence and curl magnitude of the pressure gradient for the original data, DFS and PIV-PCS with $s=0.42$ are presented in Fig. 14. Their RMS values are given in Table 2. All the gradients are estimated using a center-difference scheme. According to the governing equations, the velocity field should be divergence free, and the field of the pressure gradient should be irrotational. Because the divergence-free condition is imposed on the staggered grid for PIV-PCS, whereas DFS strictly satisfies the divergence-free condition, 


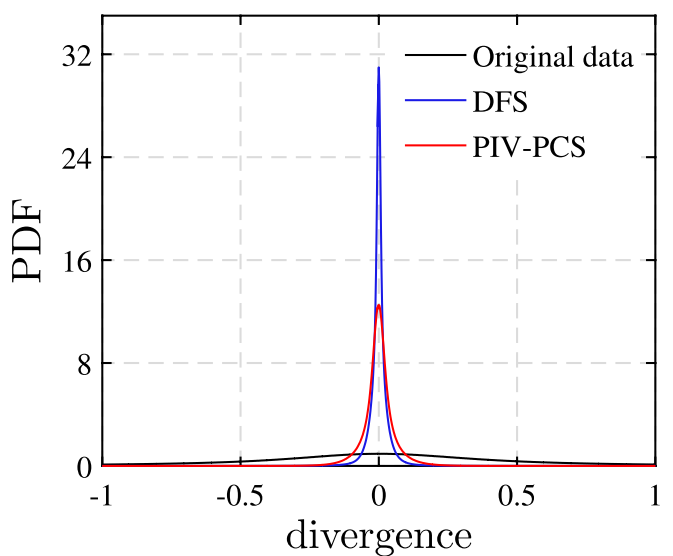

(a)

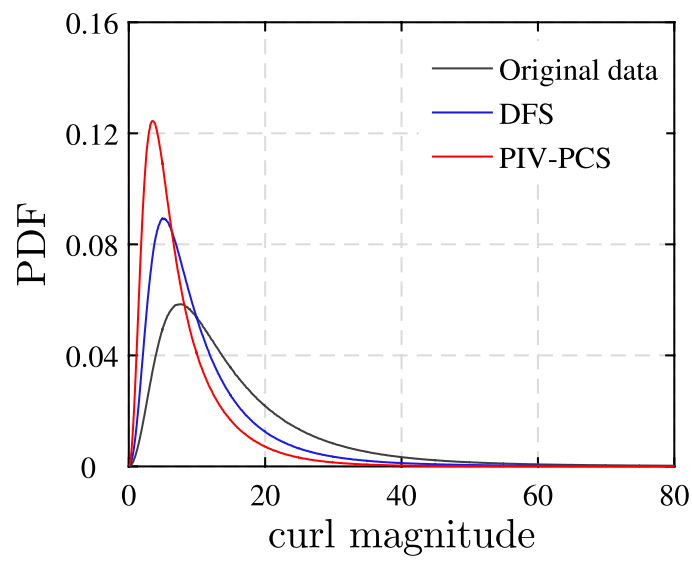

(b)

Fig. 14 PDF of the divergence (a) and curl magnitude of the pressure gradient (b) for the velocity fields of tomographic PIV, processed by DFS and PIV-PCS with $s=0.42$

Table 2 RMS of the divergence and curl magnitude of the pressure gradient for the velocity fields of tomographic PIV, processed by DFS and PIV-PCS with $s=0.42$

\begin{tabular}{lcrl}
\hline & Raw data & DFS & $\begin{array}{l}\text { PIV-PCS } \\
\mathrm{s}=0.42\end{array}$ \\
\hline RMS of divergence & 0.82 & 0.04 & 0.06 \\
RMS of curl magnitude & 17.76 & 10.65 & 6.35 \\
\hline
\end{tabular}

the PIV-PCS shows a worse performance in reducing the divergence error compared to DFS, as shown in Fig. 14a. For the curl of the pressure gradient, the PIV-PCS shows the best performance among all methods. The RMS of the curl is reduced from 17.76 for the original data to 6.35 for PIV-PCS. It is believed that the curl of the pressure gradient can be reduced to a lower level if the sampling frequency were to be high enough.

\section{Conclusions}

The full N-S equations are utilized as physical constraints to reduce the measurement error for $2 \mathrm{D}$ and $3 \mathrm{D}$ TR-PIV data in this work. The new technique proposed in this article, called PIV-PCS, uses the PCS in the projection method of CFD to solve the incompressible $\mathrm{N}-\mathrm{S}$ equations with the boundary conditions introduced by the PIV data. Because of the noisy boundary conditions, the error may be amplified by the nonlinear operators of the solver. An optimization algorithm is employed to balance the experimental and numerical parts of the objective function. The $\mathrm{N}-\mathrm{S}$ equations are decomposed into nonlinear terms, viscosity terms and pressure correction terms, and the optimization is performed with the viscosity term. Based on the numerical assessments, we also give the optimal $s$, which is a key parameter controlling the degree of optimization. In contrast to the conventional smoothing filter method, the solution from PIV-PCS can effectively smoothen out the measurement noise without introducing unwanted spatial and temporal errors.

Numerical assessments were performed on the DNS data of a 2D wake flow over a circular cylinder at a Reynolds number of 100. The PIV-PCS method was compared with DFS and another conventional filtering techniques. The proposed technique offers the best performance among all the methods when dealing with the correlated noise; however, it should be noted that PIV-PCS cannot reduce the modulation error of PIV data due to the filtered BCs. The performance with respect to pressure is more complicated. We suggest that the pressure should be solved from the optimized velocity and not directly from the pressure correction equation. The error in the pressure from the present method is also much lower than that of DFS.

The present method is validated through two real experiments: a planar PIV measurement on a $2 \mathrm{D}$ wake flow of a circular cylinder with a Reynolds number equal to that of DNS data and a tomographic PIV measurement on a 3D wake flow of a hemisphere at a Reynolds number based on a diameter of approximately 2750 . Both experiments confirm the advantages of PIV-PCS for reducing noise in terms of the velocity divergence and the curl of the pressure gradient.

Acknowledgements This work was supported by the National Natural Science Foundation of China (Nos. 11702302, 11472030 and 11572331), the Fundamental Research Funds for Central Universities (YWF-16-JCTD-A-05) and the Strategic Priority Research Program (Grant No. XDB22040104). 


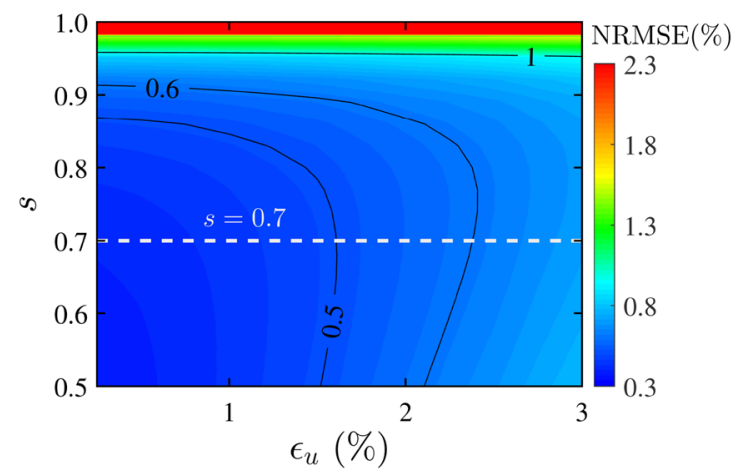

(a)

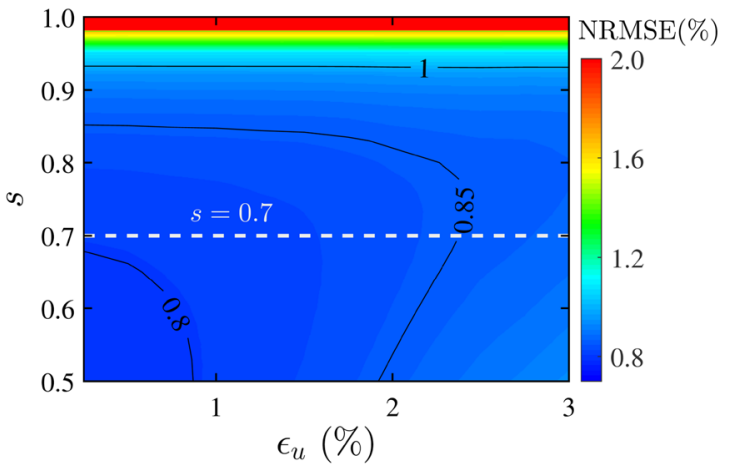

(b)

Fig. 15 The 2D contour map of the NRMSE as a function of noise level $\left(\epsilon_{u}\right)$ and $s$. The parameter $s$ is presented in the range of $0.5 \leq s \leq 1.0$. a BCs-Raw, b BCs-POD. Note that the range of the colormap is different

Fig. 16 The time evolution of the RMS difference between numerical simulation and PIVPCS for the streamwise velocity (a) and vertical velocity (b). The black, purple, green and red lines represent the results of artificial noise and PIV-PCS with $s=0.5,0.7$ and 0.97 . The noise level $\epsilon_{u}$ is set to $5 \%$. The range of the $y$-coordinate is different

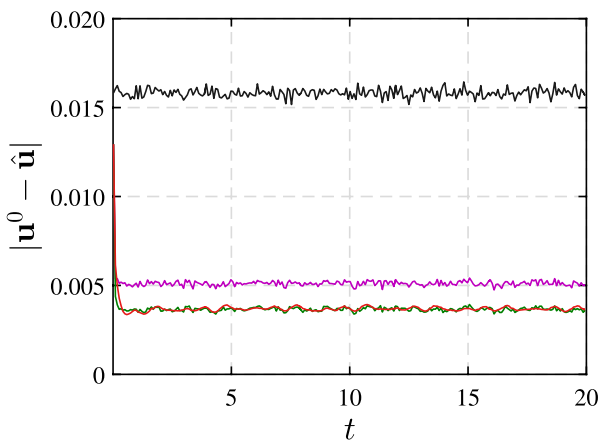

(a)

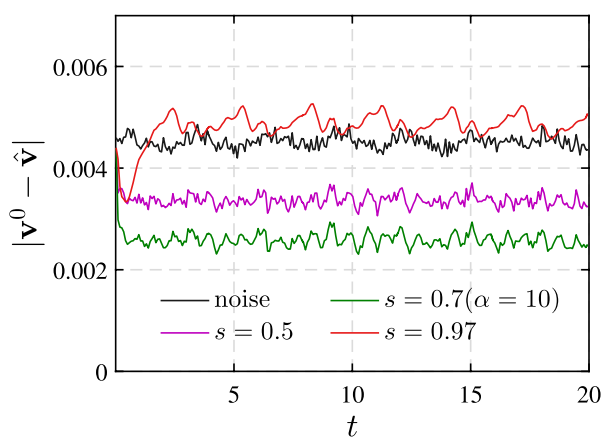

(b)

\section{Appendix}

To inspect the suitability of $\alpha=10$ at lower sampling frequencies, the 2D NRMSE map and the time history of the RMS difference under a sampling frequency of $50 \mathrm{~Hz}$ are given in Figs. 15 and 16, respectively. The illustrations in Figs. 15 and 16 are the same as that in Figs. 4 and 5, except certain values are different. The error in Fig. 15 is higher than that in Fig. 4 because of the larger time separation. The error increases with the noise level and still presents a minimum in the region of $0.7 \leq s \leq 0.8$. The optimal $s$ computed by $\alpha=10$ is equal to 0.7 , which is very close to the result from the figure. In Fig. 16, the time evolution of the error for $u$ and $v$ shows a different behavior. The PIV-PCS error of $s=0.7$ for horizontal $u$ overlaps with that of $s=0.97$; however, the error in the vertical component $v$ of $s=0.7$ is substantially smaller than that of $s=0.97$. The plot of $s=1.0$ is not shown due to the large error. From these figures, the adoption of $\alpha=10$ is found to be reasonable for estimating the optimal $s$.

\section{References}

Acarlar MS, Smith CR (1987a) A study of hairpin vortices in a laminar boundary-layer. 1. Hairpin vortices generated by a hemisphere protuberance. J Fluid Mech 175:1-41. https://doi.org/10.1017/ s0022112087000272

Acarlar MS, Smith CR (1987b) A study of hairpin vortices in a laminar boundary-layer. 2. Hairpin vortices generated by fluid injection. J Fluid Mech 175:43-83. https://doi.org/10.1017/s002211208 7000284

Adrian RJ (2007) Hairpin vortex organization in wall turbulence. Phys Fluids 19(041):301

Adrian RJ, Meinhart CD, Tomkins CD (2000) Vortex organization in the outer region of the turbulent boundary layer. J Fluid Mech 422:1-54

Andrea S, Bernhard W (2016) PIV uncertainty propagation. Meas Sci Technol 27(8):084006

Azijli I, Dwight RP (2015) Solenoidal filtering of volumetric velocity measurements using gaussian process regression. Exp Fluids 56(11):198. https://doi.org/10.1007/s00348-015-2067-7

Chorin A (1968) Numerical solution of the Navier-Stokes equations. Math Comput 22:745-762

de Kat R, van Oudheusden BW (2012) Instantaneous planar pressure determination from PIV in turbulent flow. Exp Fluids 52(5):1089_ 1106. https://doi.org/10.1007/s00348-011-1237-5

de Silva C, Philip J, Marusic I (2013) Minimization of divergence error in volumetric velocity measurements and implications for turbulence statistics. Exp Fluids 54(7):1-17. https://doi.org/10.1007/ s00348-013-1557-8 
Elsinga GE, Scarano F, Wieneke B, van Oudheusden BW (2006) Tomographic particle image velocimetry. Exp Fluids 41:933-947

Ganapathisubramani B, Longmire EK, Marusic I, Pothos S (2005) Dual-plane PIV technique to measure complete velocity gradient tensor in a turbulent boundary layer. Exp Fluids 39:222-231

Ganapathisubramani B, Longmire EK, Marusic I (2006) Experimental investigation of vortex properties in a turbulent boundary layer. Phys Fluids 18(055):105

Garcia D (2010) Robust smoothing of gridded data in one and higher dimensions with missing values. Comput Stat Data Anal 54(4):1167-1178. https://doi.org/10.1016/j.csda.2009.09.020

Garcia D (2011) A fast all-in-one method for automated post-processing of PIV data. Exp Fluids 50:1247-1259

Gesemann S, Huhn F, Schanz D, Schrder A (2016) From noisy particle tracks to velocity, acceleration and pressure fields using B-splines and penalties. In: International symposium on applications of laser techniques to fluid mechanics

Guermond JL, Minev P, Shen J (2006) An overview of projection methods for incompressible flows. Comput Methods Appl Mech Eng 195(44C47):6011-6045. https://doi.org/10.1016/j. cma.2005.10.010

Gunes H, Rist U (2007) Spatial resolution enhancement/smoothing of stereo-particle-image-velocimetry data using proper-orthogonaldecompositionlbased and Kriging interpolation methods. Phys Fluids 19:064101-064119

Higham JE, Brevis W, Keylock CJ (2016) A rapid non-iterative proper orthogonal decomposition based outlier detection and correction for PIV data. Meas Sci Technol 27(12):125303. https://doi. org/10.1088/0957-0233/27/12/125303

John JC, Cameron VK, Barton LS, Pavlos PV (2010) Assessment of pressure field calculations from particle image velocimetry measurements. Meas Sci Technol 21(10):105401. https://doi. org/10.1088/0957-0233/21/10/105401

Liu XF, Katz J (2006) Instantaneous pressure and material acceleration measurements using a four-exposure PIV system. Exp Fluids 41(2):227. https://doi.org/10.1007/s00348-006-0152-7

Lynch KP, Scarano F (2015) An efficient and accurate approach to MTE-MART for time-resolved tomographic PIV. Exp Fluids 56(3):1-16

Lynch K, Prbsting S, Scarano F (2014) Temporal resolution of timeresolved tomographic PIV in turbulent boundary layers. In: 17th international symposium on applications of laser techniques to fluid mechanics

McClure J, Yarusevych S (2017) Optimization of planar PIV-based pressure estimates in laminar and turbulent wakes. Exp Fluids 58(5):62. https://doi.org/10.1007/s00348-017-2337-7

Novara M, Scarano F (2012) Performances of motion tracking enhanced Tomo-PIV on turbulent shear flows. Exp Fluids 52(4):1027-1041

Novara M, Batenburg KJ, Scarano F (2010) Motion tracking-enhanced MART for tomographic PIV. Meas Sci Technol 21(3):18. https:// doi.org/10.1088/0957-0233/21/3/035401

Okuno T, Sugii Y, Nishio S (2000) Image measurement of flow field using physics-based dynamic model. Meas Sci Technol 11(6):667-676. https://doi.org/10.1088/0957-0233/11/6/309

Oudheusden BWV (2013) PIV-based pressure measurement. Meas Sci Technol 24(3):032001. https://doi.org/10.1088/09570233/24/3/032001

Pan Z, Whitehead J, Thomson S, Truscott T (2016) Error propagation dynamics of PIV-based pressure field calculations: how well does the pressure poisson solver perform inherently? Meas Sci Technol 27(8):084012. https://doi.org/10.1088/09570233/27/8/084012
Raffel M (2007) Particle image velocimetry: a practical guide, 2nd edn. Springer, New York

Scarano F (2002) Iterative image deformation methods in PIV. Meas Sci Technol 13(1):R1-R19

Schneiders JFG, Scarano F (2016) Dense velocity reconstruction from tomographic PTV with material derivatives. Exp Fluids 57(9):122. https://doi.org/10.1007/s00348-016-2225-6

Schanz D, Schröder A, Gesemann S (2014) 'Shake The Box'-a 4D PTV algorithm: accurate and ghostless reconstruction of Lagrangian tracks in densely seeded flows. In: 17th international symposium on applications of laser techniques to fluid mechanics

Schanz D, Gesemann S, Schrder A (2016) Shake-the-box: Lagrangian particle tracking at high particle image densities. Exp Fluids 57(5):1-27. https://doi.org/10.1007/s00348-016-2157-1

Schneiders JG, Dwight R, Scarano F (2014) Time-supersampling of 3D-PIV measurements with vortex-in-cell simulation. Exp Fluids 55(3):1-15. https://doi.org/10.1007/s00348-014-1692-x

Schneiders JFG, Dwight RP, Scarano F (2015) Tomographic PIV noise reduction by simulating repeated measurements. In: International symposium on particle image velocimetry

Sciacchitano A, Dwight R, Scarano F (2012a) Navier-Stokes simulations in gappy PIV data. Exp Fluids 53(5):1421-1435. https://doi. org/10.1007/s00348-012-1366-5

Sciacchitano A, Scarano F, Wieneke B (2012b) Multi-frame pyramid correlation for time-resolved PIV. Exp Fluids 53(4):1087-1105. https://doi.org/10.1007/s00348-012-1345-x

Smits AJ, McKeon BJ, Marusic I (2011) High-Reynolds number wall turbulence. Ann Rev Fluid Mech 43(1):353-375. https://doi. org/10.1146/annurev-fluid-122109-160753

Strang G (2007) Computational science and engineering. WellesleyCambridge Press, Wellesley

Suzuki T (2012) Reduced-order Kalman-filtered hybrid simulation combining particle tracking velocimetry and direct numerical simulation. J Fluid Mech 709:249-288. https://doi.org/10.1017/ jfm.2012.334

Suzuki T, Ji H, Yamamoto F (2006) Unsteady PTV velocity field solved with DNS, American Institute of Aeronautics and Astronautics. In: Fluid Dynamics and Co-located Conferences. https://doi. org/10.2514/6.2006-3450

Suzuki T, Ji H, Yamamoto F (2009) Unsteady PTV velocity field past an airfoil solved with DNS: Part 1. Algorithm of hybrid simulation and hybrid velocity field at $R e \approx 10^{3}$. Exp Fluids 47(6):957976. https://doi.org/10.1007/s00348-009-0691-9

Tang CX, Sun WF, He HY, Li HQ, Li EB (2017) Spurious PIV vector detection and correction using a penalized least-squares method with adaptive order differentials. Exp Fluids 58(7):81. https://doi. org/10.1007/s00348-017-2350-x

Témam R (1969) Sur l'Approximation de la Solution des Équations de Navier-Stokes par la Méthode des Pas Fractionnaires (II). Arch Ration Mech Anal 33(5):377-385. https://doi.org/10.1007/BF002 47696

van Gent PL, Michaelis D, van Oudheusden BW, Weiss P, de Kat R, Laskari A, Jeon YJ, David L, Schanz D, Huhn F, Gesemann S, Novara M, McPhaden C, Neeteson NJ, Rival DE, Schneiders JFG, Schrijer FFJ (2017) Comparative assessment of pressure field reconstructions from particle image velocimetry measurements and Lagrangian particle tracking. Exp Fluids 58(4):33. https://doi.org/10.1007/s00348-017-2324-z

Venturi D, Karniadakis GE (2004) Gappy data and reconstruction procedures for flow past a cylinder. J Fluid Mech 519:315-336

Wang ZY (2017) PIV based pressure reconstruction technique and its application. PhD thesis, Beihang University, Beijing, China

Wang SZ, Zhang X (2011) An immersed boundary method based on discrete stream function formulation for two- and three-dimensional 
incompressible flows. J Comput Phys 230(9):3479-3499. https:// doi.org/10.1016/j.jcp.2011.01.045

Wang HP, Gao Q, Feng LH, Wei RJ, Wang JJ (2015) Proper orthogonal decomposition based outlier correction for PIV data. Exp Fluids 56(2):1-15. https://doi.org/10.1007/s00348-015-1894-x

Wang CY, Gao Q, Wang HP, Wei RJ, Li T, Wang JJ (2016a) Divergence-free smoothing for volumetric PIV data. Exp Fluids 57(1):1-23. https://doi.org/10.1007/s00348-015-2097-1

Wang HP, Gao Q, Wei RJ, Wang JJ (2016b) Intensity-enhanced MART for tomographic PIV. Exp Fluids 57(5):1-19. https://doi. org/10.1007/s00348-016-2176-y

Wang ZY, Gao Q, Wang CY, Wei RJ, Wang JJ (2016c) An irrotation correction on pressure gradient and orthogonal-path integration for PIV-based pressure reconstruction. Exp Fluids 57(6):16. https ://doi.org/10.1007/s00348-016-2189-6
Wang CY, Gao Q, Wei RJ, Li T, Wang JJ (2017) Weighted divergence correction scheme and its fast implementation. Exp Fluids 58(5):14. https://doi.org/10.1007/s00348-017-2307-0

Westerweel J, Scarano F (2005) Universal outlier detection for PIV data. Exp Fluids 39(6):1096-1100

Zhou J, Adrian RJ, Balachandar S, Kendall TM (1999) Mechanisms for generating coherent packets of hairpin vortices in channel flow. $\mathrm{J}$ Fluid Mech 387:353-396

Publisher's Note Springer Nature remains neutral with regard to jurisdictional claims in published maps and institutional affiliations. 